\title{
Eye movements during search and detection in comparative visual search
}

\author{
ADAM J. GALPIN \\ University of Manchester, Manchester, England \\ and \\ GEOFFREY UNDERWOOD \\ University of Nottingham, Nottingham, England
}

\begin{abstract}
Motivated by the fact that previous visual memory paradigms have imposed encoding and retrieval constraints, the present article presents two experiments that address how observers allocate eye movements in memory and comparison processes in the absence of constraints. A comparative visual search design (Pomplun, Sichelschmidt, et al., 2001) was utilized in which observers searched for a difference between two images presented side by side. Robust time course effects were obtained, whereby search was characterized by brief fixations and a high proportion of comparative saccades. Then, upon target detection, fixations were extended, more comparative saccades were elicited, and the search focus was narrowed. The saliency and presence of differences did not guide attention, and detection was contingent upon direct fixation of the targets. The results indicate that, when full control is given, observers adopt a strategy that cuts down on memory usage in favor of restricted encoding and active scanning.
\end{abstract}

Visual acuity for scene detail declines as the distance from the fovea increases, which means that eye movements are necessary to bring regions into foveal vision if they require in-depth scrutiny. When inspecting visual stimuli, observers typically gaze at two or three different details per second, and this period, when the eyes are relatively still, is referred to as a fixation. To move the point of fixation to a new area of interest involves a saccadic eye movement, during which the eye is rotated rapidly in its socket and visual input is suppressed (Matin, 1974). The pioneering work of Yarbus (1967) has demonstrated that the patterns of fixations and saccades involved in picture viewing depends on the current goals of the observer; thus, eye movements have become a useful tool in vision research for inferring underlying mental states from overt behavior. In particular, eye movements become useful if a strategy or tendency is adjusted over the time course of an activity, in which instance they can be used to postulate frameworks and stages for processing. For example, Just and Carpenter (1976) analyzed the scan paths of observers making same/different judgments on rotated figures and used the frequency and position of fixations that switch between the figures to divide trials into stages of search, comparison, and confirmation. In addition to the location of fixations, changes in fixation duration are useful markers of a change in strategy or processing, since they in-

Correspondence concerning this article should be addressed to A. J. Galpin, Department of Psychology, University of Manchester, Coupland Street, Manchester M13 9PL, England (e-mail: adam.galpin@ manchester.ac.uk). crease with the difficulty of the foveal task (Gould, 1967, 1973; Hooge \& Erkelens, 1998; Jacobs, 1986; Jacobs \& O'Regan, 1987) and vary between processes such as encoding and comparing (Gould, 1967; Gould \& Peeples, 1970).

The present article addresses the ways in which observers employ eye movements to encode visual information into memory. We utilized a spot-the-difference task, in which observers must memorize details from one image before directing gaze at a second image to perform a comparison. This design falls within the broader realm of visual memory tasks; thus, a detour through some of the literature is required before the specific aims of the article are presented. Early experiments concerned with long-term picture memory suggested that pictorial stimuli could be remembered well and over long periods of time (Shepard, 1967; Standing, Conezio, \& Haber, 1970). However, a large body of more recent work on the phenomenon referred to as change blindness (Rensink, O'Regan, \& Clark, 1997) suggests that observers are not aware of much of the detail of perceptually present stimuli, calling into question how rich their memory can be for stimuli that have been removed. Change blindness refers to an inability to detect alterations in visual stimuli and is easily induced by a visual diversion that either covers the change or draws attention from the change location. One widely used method, known as the flicker paradigm (developed by Rensink et al., 1997), presents pairs of alternating images containing a change, separated by a brief interval, during which the change is made. Observers often search for lengthy periods before they can detect what is different between the images, demonstrating that a complete and 
detailed representation of each image is not readily available for comparison. The flicker mimics the lack of visually processed information during a saccade, and in fact, changes made during real saccadic eye movements also go unnoticed (Henderson \& Hollingworth, 2003; Hollingworth \& Henderson, 2002; McConkie \& Currie, 1996).

The study of visual memory with eye movements has enjoyed a long tradition. Many early experiments were concerned with eye movements and long-term picture memory (Loftus, 1972; Nelson \& Loftus, 1980), whereas more recent work has investigated the memory representations that are formed online during scene viewing (e.g., Henderson \& Hollingworth, 2003; Hollingworth \& Henderson, 2002; Hollingworth, Schrock, \& Henderson, 2001; Zelinsky, 2001) and the search of scenes prompted by the evaluation of accompanying text (Underwood, Jebbett, \& Roberts, 2004). One key finding that has emerged from these studies is that memory for pictorial detail is highly contingent upon fixation behavior. First, when observers are prevented from making eye movements, memory performance is impaired (Hollingworth et al., 2001; Irwin \& Zelinsky, 2002). Second, when eye movements are permitted, scene details that have been fixated show a recall or recognition advantage (Hollingworth \& Henderson, 2002; Irwin \& Zelinsky, 2002; Melcher \& Kowler, 2001; Nelson \& Loftus, 1980; Underwood, Chapman, Berger, \& Crundall, 2003).

These results favor an interpretation whereby direct fixation on an object plays a major role in storage of that object in both short- and long-term memory. However, some evidence also supports a role for the encoding of scene details that have appeared only in the visual periphery. In the experiments of Hollingworth et al. (2001), the change detection rate was high $(>90 \%)$ even when eye movements were prevented. Irwin and Zelinsky (2002) also found recognition of objects to exceed chance performance when no eye movements were allowed across a study scene. Zelinsky (2001) found evidence for peripheral discrimination in scan paths in a change detection flicker task. On many trials, the scan paths came to an abrupt stop in order that a changed object might be fixated, suggesting that the change had been detected while gaze was directed elsewhere. Although observers exhibited a strong preference to fixate objects upon detection, the scan paths suggested that these fixations merely served a confirmatory function.

Furthermore, it would appear that peripheral information is also available during the recognition process. Parker (1978) tested memory for a simple line drawing containing six objects, using a series of same/different judgments based on similar stimuli. Changes were made by removing, changing the size of, or replacing a single object. Eye movement data from the recognition phase revealed that changed objects were looked at, on average, over one fixation earlier than unchanged objects. On the basis of the distance between consecutive objects, Parker estimated that useful information is extracted from at least $6.5^{\circ}$ into the periphery. Parker's results have not gone unchallenged, however. Henderson, Williams, Castelhano, and Falk (2003) tested the claim that changes are detected in the periphery and fixated sooner than unchanged objects. Although changed objects were fixated sooner, the saccades that directed gaze at these objects were no longer than those for unchanged objects, suggesting that peripheral processing is limited and can occur only once gaze is within close proximity. On balance, however, the bulk of evidence suggests that scene details beyond the current focus of gaze can be used for both encoding and comparative purposes.

The majority of experiments described above assessed memory for a scene that was no longer perceptually present. Although this is the most direct way to probe memory representations, it also presents a scenario at odds with most real-life situations. As an observer interacts with the visual world, details are available as and when needed; thus, there is little necessity for a rich, detailed representation (O'Regan, 1992). In the long-term memory experiments (e.g., Loftus, 1972; Nelson \& Loftus, 1980), participants had only one chance to encode a stimulus, with further limits placed on the time available to later retrieve this representation and compare it with the perceptually present stimuli. The flicker paradigm utilized by Zelinsky (2001) and Hollingworth et al. (2001) at least affords multiple looks between images, although constraints are still imposed in terms of how long each image is presented: Essentially, control is taken away from the observer. Therefore, in the present experiments, we asked about the pattern of eye movements in a visual memory task when observers were free to set their own inspection parameters. This is particularly interesting, since some of the studies described above found strategic adaptations in fixation behavior to be influenced by task constraints. For instance, Nelson and Loftus found that the ability to recognize pictures on the basis of objects viewed in the visual periphery was present only when the study scene had been viewed under longer exposure durations. It was suggested that this could reflect strategic adaptations in the functional field under different viewing constraints. When there is more time to look, participants widen the field from which information is obtained. This effect may have been mediated by longer fixations when participants were given more time to look, although Nelson and Loftus did not perform this comparison. However, evidence does come from Melcher and Kowler (2001), who found that participants demonstrated a preference for longer fixation durations during longer exposures, possibly because they were scanning more frantically when the pictures were presented briefly, in an attempt to fixate more objects. This result could be taken to suggest that the scanning behavior reflects a natural desire to bring objects into foveal vision. Zelinsky's eye movement study of performance in a flicker task emphasized a different aspect of behavior. His scan path analysis suggested that participants did not favor the obvious strategy of consecutively comparing preand postchange objects. Instead, they apparently scanned the display as if it was a static image, meaning that detection often occurred once gaze had left the targets. These results highlight two potential ways in which behavior is 
constrained by aspects of experimental design. First, limits set by exposure duration influence scanning rate and, possibly, the functional field for information acquisition. Second, tasks such as the flicker task may violate certain assumptions that the observer has about the environment. Simons (2000) has suggested that an underlying assumption of the cognitive system is that things in the visual world do not change, and this may be reflected by search in Zelinsky's task, which did not appear to accommodate the presence of the flicker.

\section{Comparative Visual Search}

To investigate perceptual strategies in the absence of constraints, we chose a task that involved a memory component yet did not impose viewing restrictions in terms of encoding and comparison processes. We placed two virtually identical images side by side and asked observers to search between them for a difference. This task is commonly referred to as spot the difference, although we shall adopt the term comparative visual search (CVS, hereafter) from previous workers who have used this design (Pomplun, Reingold, \& Shen, 2001; Pomplun \& Ritter, 1999; Pomplun, Sichelschmidt, et al., 2001). To be sure, searching for a difference between simultaneously presented pictures is a memory task: Sensory information is tied to spatial location (Phillips, 1974) and cannot survive an eye movement (Irwin, 1991). Indeed, Irwin's experiments demonstrated that the visual information carried across a saccadic eye movement does not exceed visual shortterm memory capacity, and the two are probably served by the same mechanisms. Therefore, in order to compare the details of one picture with those of another, information must be encoded into memory from one image, before an eye movement allows comparison between the stored representation and the perceptually present corresponding image. The task is functionally similar to the continually alternating images presented using the flicker design. However, we wondered how observers would choose to coordinate memory and comparison processes in the $a b$ sence of constraints.

Work by Pomplun and colleagues has gone some way toward addressing this issue, although the emphasis was on search processes, rather than on those involved in memory. Pomplun, Sichelschmidt, et al. (2001) investigated comparative search for color and form differences in arrays of shapes, finding that the strategy that best fitted the experimental data was a clustering strategy, whereby working memory appeared to be maximally utilized in order to encode as much detail as possible before a saccade was elicited to perform a comparison with the corresponding image. This was evidenced by an effect of the number of surrounding objects on fixation duration, suggesting that multiple objects were chunked. Likewise, the number of successive fixations before a comparison was elicited also decreased as object density increased. This indicates that two dependent measures that are sensitive to memory constraints in CVS are fixation duration and the number of fixations within a single image before a comparison is elicited.
In terms of the global characteristics of the search, Pomplun, Sichelschmidt, et al. (2001) found that eye movements afforded the temporal division of trials into a lengthy search and comparison phase, followed by a brief detection and verification phase. The latter stage was operationally defined as occurring when gaze landed within approximately $2^{\circ}$ of visual angle of the target objects and a response was registered within $2 \mathrm{sec}$. Typically, fixations were longer within the detection and verification phase, and the number of comparative saccades between the images increased, whereas the size of the saccades directed within an image decreased. These findings were interpreted as demonstrating an increase in concentration and a decrease in the size of the image region under attentional focus after detection of a suspicious image region.

\section{The Present Experiments}

The time course of search behavior from trial onset to offset provides a framework within which to structure the more detailed aspects of search. Therefore, as our starting point, we chose to test Pomplun's claim that detection is served by characteristic oculomotor behavior. The interpretation holds intuitive appeal, yet there may be other reasons why the last few fixations were found to exhibit eye movement characteristics different from those at the beginning of the trial. Specifically, both fixation duration and saccade length have been shown to vary over the whole time course of image viewing in scene perception (see, e.g., Antes, 1974; Buswell, 1935). Irwin and Zelinsky (2002), for example, reported a steady increase in fixation duration over a 15-fixation-long study period of a simple scene containing toys in a crib. The same trends have also been observed in the search literature. Scinto, Pillalamarri, and Karsh (1986) found a general trend for an increase in fixation duration and a decrease in saccade size when successive groups of 10 fixations were analyzed from an extended search task, indicating that such adjustments begin well before the onset of target detection. Scinto et al. interpreted these findings as reflecting strategic modifications to so far unsuccessful searches. The implications for the conclusions drawn by Pomplun, Sichelschmidt, et al. (2001) are that if only the last few fixations that occur during detection were analyzed and compared with the initial portion of a trial, apparent differences could be due to sampling the end point after continuous adjustments over the course of the trial.

To answer the question about whether oculomotor variables in CVS are adjusted following detection, or whether this adjustment occurs throughout the whole of the trial, the present experiments assessed eye movements from trial onset to response (Experiment 1), and also included same trials to view trends in the absence of detection (Experiment 2). A new method was developed that assessed time course by dividing each trial into four time quartiles based on the number of eye movement events (i.e., fixations or saccades). In addition to this global overview of search behavior, a more fine-grained analysis was conducted of the last five fixations preceding response. This was added in order to assess whether eye movements 
within the detection phase vary with the difficulty of the discrimination. No differences between color and form were observed within the detection and verification period by Pomplun and colleagues, but these dimensions were not selected to vary with ease of discrimination. The present experiments extended this work by using complex arrays of real-world objects (toys) containing a variety of difference types assumed to vary in terms of the ease with which they can be discriminated (additions/deletions [ADs], object substitutions [OSs], location changes [LCs], and object flips [OFs]).

We were also interested in how gaze interacted with the two target regions that made up each difference. Do observers display an object-by-object comparison strategy, or is information from several objects encoded before a comparison is elicited? A detailed analysis of target fixation behavior was conducted for trials in which the difference was detected, but also for trials in which the difference was missed. A related question concerns whether the peripheral discrimination found in Parker's (1978) study might be present when the present design is used. Consider the different potential search patterns in the CVS task. At one extreme, a single fixation on each image might be used to detect the difference, perhaps followed by a long saccade to the targets for confirmation. Short-term memory constraints make this option unlikely, although it is possible that several objects could be encoded within each fixation, allowing for earlier fixation of salient differences. At the other extreme, participants may choose to encode only one or two objects with each visit - in which case, differences might not be detected until gaze is close to the target objects. After Parker (1978) and Henderson et al. (2003), we employed two measures to test peripheral guidance: the number of fixations before the targets are fixated and the size of the saccade that directs gaze at the targets. Experiment 1 assessed whether salient differences (object deletions) were fixated sooner than more subtle discriminations (OSs and LCs). If the mere presence of an object is peripherally detected in one image and its absence is noted in the other, these targets might be fixated sooner. Experiment 2 assessed whether target objects containing a difference were fixated sooner than the same objects in matched same stimuli that did not contain a difference. This was done in order to assess whether the presence of a difference was detected peripherally and, thus, attracted earlier fixation.

\section{EXPERIMENT 1}

\section{Method}

Participants. Thirty (23 of them female) members of the staff and student population of the University of Nottingham participated in exchange for $£ 4$. All had normal or corrected-to-normal vision.

Stimuli. The stimuli consisted of pairs of color photographs arranged side by side on a white background, separated by a distance of $2.6^{\circ}$ of visual angle at the viewing distance of $70 \mathrm{~cm}$. Each picture subtended $12.5^{\circ} \times 9.4^{\circ}$, and corresponding points between the two images subtended $15.1^{\circ}$ of visual angle. All the photographs depicted arrays of toys scattered on a white tablecloth. Each photograph contained either 10 or 11 objects (evenly distributed among the conditions) from 12 categories of toys that could naturally be found in a child's play area. The objects making up each category were intended to have the same basic-level category heading (i.e., cars, dinosaurs, marbles).

The photographs in each pair differed in one of three ways (with 16 examples of each). First, an object present in one image might be absent in the other (AD). Second, an object could be substituted for another object from the same category, chosen to be of similar appearance in terms of size and color (OS). Third, an object could be displaced horizontally in one of the images (LC). Examples of each of the difference type conditions can be seen in Figure 1. These three difference type conditions were chosen for the ease with which they could be discriminated; the removal of an object is predicted to be easier to detect than an $\mathrm{LC}$, which in turn is predicted to be easier than OS (these predictions are based on findings from the change detection literature; see Rensink, 2002; Simons, 1996). In addition, 16 filler items in which part of an object could be removed, be rotated, be shifted, or change color were included in order to reduce the chance that participants might form expectations for the difference types. Finally, eight catch trials containing no difference were included in order to identify any participants who might be guessing. The pictures originally came from an experiment investigating perceptual categorization; half contained objects from mixed categories, and half contained objects from the same category. The uniformity of the objects was found to have no influence over search variables and is, therefore, not discussed or analyzed in the present investigation.

Apparatus. Images were displayed on a $36 \times 27 \mathrm{~cm}$ monitor with a resolution of 1,024 $\times 768$ pixels. An SMI EyeLink system was used to record eye movements. In the EyeLink system, a headmounted high-speed camera takes an image of the right pupil every $4 \mathrm{msec}$ and affords spatial accuracy of greater than $0.5^{\circ}$. To detect saccadic eye movements, an online parser computes the distance between the eye at consecutive samples and implements a velocity threshold of $30^{\circ} / \mathrm{sec}$, an acceleration threshold of $4,000^{\circ} / \mathrm{sec}^{2}$, and a saccade motion threshold of $0.15^{\circ}$. The resting point between saccades is defined as a fixation.

Design. The within-participants variable of difference type contained three (AD, LC, and OS) experimental conditions containing 16 items each. Reaction time, number of fixations, and accuracy were analyzed using a one-way ANOVA, to provide a general indication of the ease with which these differences could be detected. The way in which gaze interacted with the targets was also of interest. In addition to looking at the number of target-directed fixations, we used two measures to assess whether larger differences were fixated earlier. The first was the number of fixations before the first target fixation. The second was the distance that a saccade moved from corresponding points in order to fixate a target. The latter measure is not simply saccade size, since a target may be fixated following either a short saccade directed within an image or a long saccade that crossed between images. Instead, it made sense to consider the distance between corresponding points in the two images (e.g., the distance between a pair of objects; $15.1^{\circ}$, as shown in Figure 1). Subtracting this distance from the horizontal $x$-coordinate of all saccades that landed on the right-hand image effectively allowed the display to be treated as if it were a single-image display. This offered a measure of how far saccades were directed away from the current search region, whether within the same image or away from the corresponding region in the other image. A third measure of interest concerned the number of fixations that occurred between consecutive views of the two targets. Because a pair of target objects could be fixated multiple times within a trial and with a different number of intervening fixations on each occasion, for the sake of consistency this analysis always used the episode with the least number of 

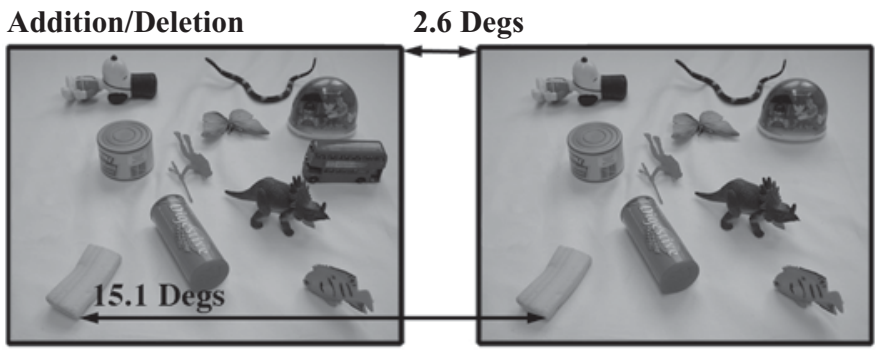

Object Substitution

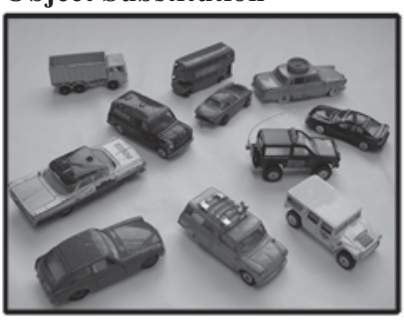

Location Change

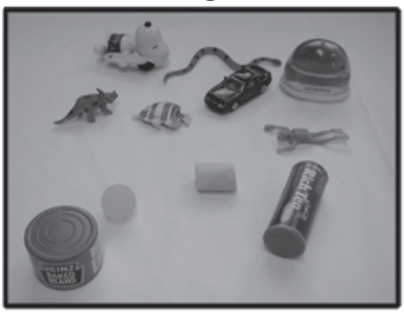

Figure 1. Examples of the stimuli from Experiment 1. Note that the actual pictures were shown in color. Furthermore, note that whether the objects were from the same or different categories was evenly distributed among conditions.
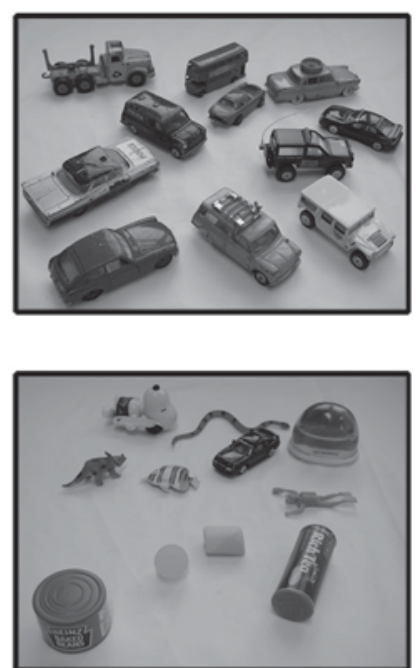

\section{Results}

The participants took, on average, $6.6 \mathrm{sec}$ to correctly spot a difference, although this varied somewhat between participants, with mean time to spot differences ranging from 3.6 to $11.5 \mathrm{sec}(S D=1.7)$. More variable was the time taken to correctly make a same response to catch trials $(M=17.8 \mathrm{sec}, S D=7.2)$, with average response times for the participants ranging from 5.7 to $35.5 \mathrm{sec}$. The participants proved their concentration by responding correctly to the catch trials. Only one responded incorrectly to more than two; thus, it was decided that no participants should be removed on the basis of errors. All further analyses discussed are based on data without the catch trials, filler trials, or incorrect responses. For eye movement analysis, fixations lasting less than $100 \mathrm{msec}$ were removed from the data set. One participant, whose eye tracking was consistently poor, was removed from all eye-tracking analyses.

Accuracy scores (Figure 2A) for the three difference type conditions were analyzed using a one-way ANOVA with three levels. A significant main effect was found for difference type $[F(2,58)=11.418, p<.0001]$. Post hoc Tukey's tests revealed that this effect was due to superior detection accuracy for $\mathrm{AD}(98 \%)$ over OS $(88 \% ; q=5.19$, $p<.01)$ and $\mathrm{LC}(85 \% ; q=6.34, p<.001)$. No difference 
A) Accuracy

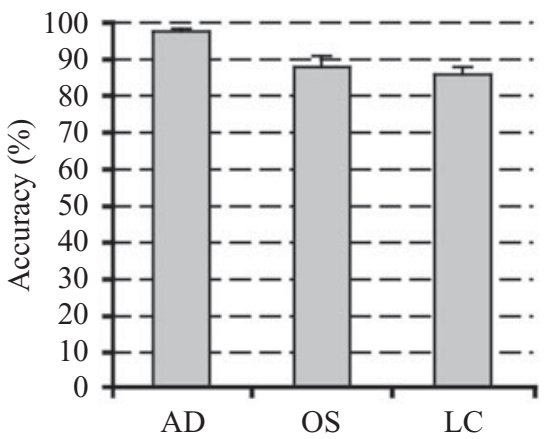

C) Number of Fixations

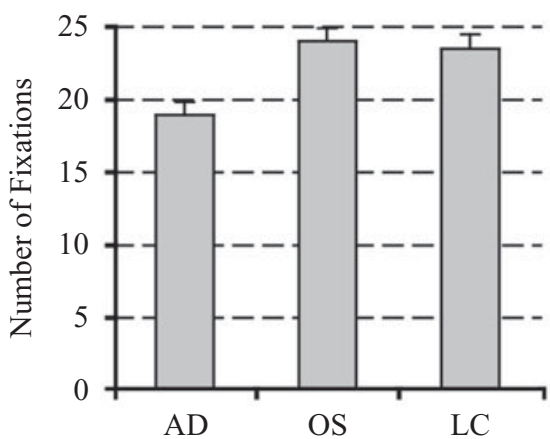

B) Reaction Time

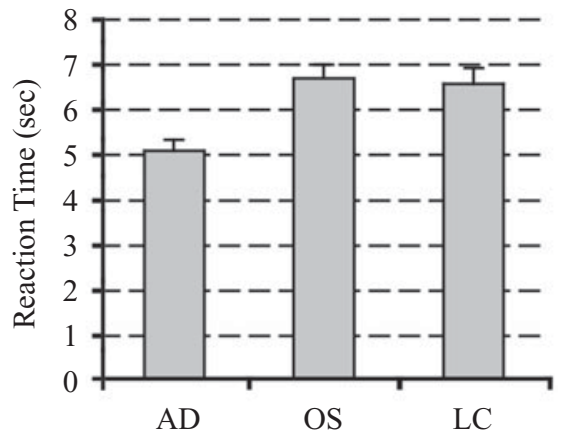

D) Number of Target Fixations

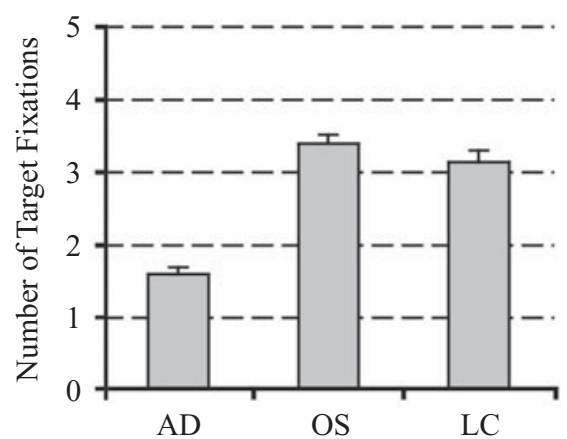

Figure 2. Overview of the results from Experiment 1. Charts show (A) accuracy, (B) mean reaction times, $(C)$ the average number of fixations, and (D) the average number of target fixations. In all cases, the error bars represent the standard error of the mean. AD, addition/ deletion; OS, object substitution; LC, location change.

existed between OS and LC. For the reaction time analysis (Figure 2B), data points were removed as outliers if they exceeded a cutoff of three standard deviations above or below the grand mean, resulting in the loss of responses greater than $22.3 \mathrm{sec}(1.46 \%$ of the responses). Means for the remaining data were then square root transformed to account for positive skew. A significant main effect was found for difference type $[F(2,58)=21.127, p<.0001]$, due to faster reaction times for $\mathrm{AD}(5.1 \mathrm{sec})$ than for OS $(6.7 \mathrm{sec} ; q=8.31, p<.001)$ and LC (6.6 sec; $q=7.56$, $p<.001)$. No difference existed between OS and LC. Unsurprisingly, the same trends existed for the number of fixations (Figure 2C), with fewer fixations being required for AD (19) than for OS $(24 ; p<.001)$ and for AD than for LC $(23.5 ; p<.001)$. The analyses for accuracy, reaction time, and number of fixations show a consistent story in terms of the ease with which the difference can be detected: Deletions are easiest, and OSs and LCs do not differ from each other.

Target fixations. To assess whether fixations fell on the targets, coordinates for target areas were derived by drawing imaginary boxes with a distance of five pixels from the extreme top, bottom, left, and right edges. Often, more than one box was necessary to encompass objects not conveniently rectangular. The blank area in the AD condition was assigned coordinates based on the position of the corresponding present object. The number of target fixations in each condition can be seen in Figure 2D. An effect of the average number of target fixations $[F(2,56)=$ $95.202, p<.0001]$ was due to fewer target fixations in the $\mathrm{AD}$ condition (1.6) than in the OS condition $(3.4 ; q=$ $18.02, p<.001)$ and the LC condition $(3.1 ; q=15.5$, $p<.001)$. Presumably, much of the effect here occurred because the observers were reluctant to fixate the blank area of space left by the removal of a target in the AD condition. No difference existed between the OS and the LC conditions.

We also wondered whether target fixation behavior might be able to account for the trials in which the difference was missed. An obvious possibility was that the target objects were simply not fixated as often in these trials. However, no simple relationship existed between detection and the number of target fixations. There were fewer target fixations in the miss trials than correct detections in the $\mathrm{AD}$ condition ( 0.9 , as compared with 1.6) and the OS condition (3.3, as compared with 3.4), but there were actually more target fixations in the miss trials in the LC condition (4.0, as compared with 3.1). A more complex hypothesis concerns whether both targets were fixated and how many shifts of attention occurred between consecutive views of both targets. To assess this possibility, we measured the number of fixations that occurred 
between consecutive views of the two targets on each trial. If the targets had been fixated multiple times, we took the least number of fixations between consecutive views. The results of this analysis can be viewed in Figure 3, which depicts target fixation behavior separately for each difference type, correct detections, and misses. The first two categories on the $x$-axis represent, respectively, those trials in which the target was not fixated at all and those instances in which only one of the two targets was fixated (this does not mean that there was only one target fixation, for the same target may have been fixated multiple times). The remaining categories show the least number of nontarget fixations that occurred between consecutive views of both targets. The $y$-axis shows the proportion of trials falling into each category. As can be seen, the majority of correct detections (black bars) in the OS and LC conditions were resolved only after both targets had been consecutively viewed (.61 and .53, respectively) or viewed with either one or two nontargets intervening $(\mathrm{OS}=.20, \mathrm{LC}=$ .17 , respectively). Only on a few occasions was detection resolved with no target fixations or fixation of only one target. A trend existed in both these conditions for fewer consecutive target fixations to occur in the misses (white bars) and for more instances when only one (or no) target was fixated. Although this trend was not overwhelming, the fact that it was consistent across conditions suggests that some of the misses occurred because comparisons between the targets did not happen soon enough or at all. The AD condition presents a different story, although this is again likely to have been due to a reluctance to fixate a blank space. In fact, the few errors in this condition seem more straightforward to explain: .42 occurred because there were no target fixations at all.

Peripheral guidance. The fact that the large majority of trials involved at least one target fixation allows an analysis of how the eyes were guided to the first target fixation. The reaction time and number of fixations analyses (above) show an advantage for the AD condition, but this may not have been because attention was directed to these targets earlier; rather, it could be that differences were less likely to be missed on an initial inspection or because discrimination took less time once attention reached the targets. Therefore, the number of fixations before the target was fixated was calculated for each difference type. However, as is apparent from Figure 4A, no effect was found $(F<1)$. The second measure assessing peripheral guidance was the distance moved from corresponding points to direct gaze to the first target fixation. It might be expected that the differences in the AD condition would be detected from a further distance than the other, more subtle differences. However, as is shown in Figure 4B, an effect of distance moved $[F(2,56)=12.104, p<.0001]$ was, in fact, due to a smaller distance in the AD condition $\left(2.6^{\circ}\right)$ than in both the OS condition $\left(3.0^{\circ} ; q=4.51, p<\right.$ $.01)$ and the LC condition $\left(3.2^{\circ} ; q=6.84, p<.001\right)$. The fact that these distances are small, and similar, suggests that peripheral guidance does not play much of a role in drawing attention to the location of the differences.
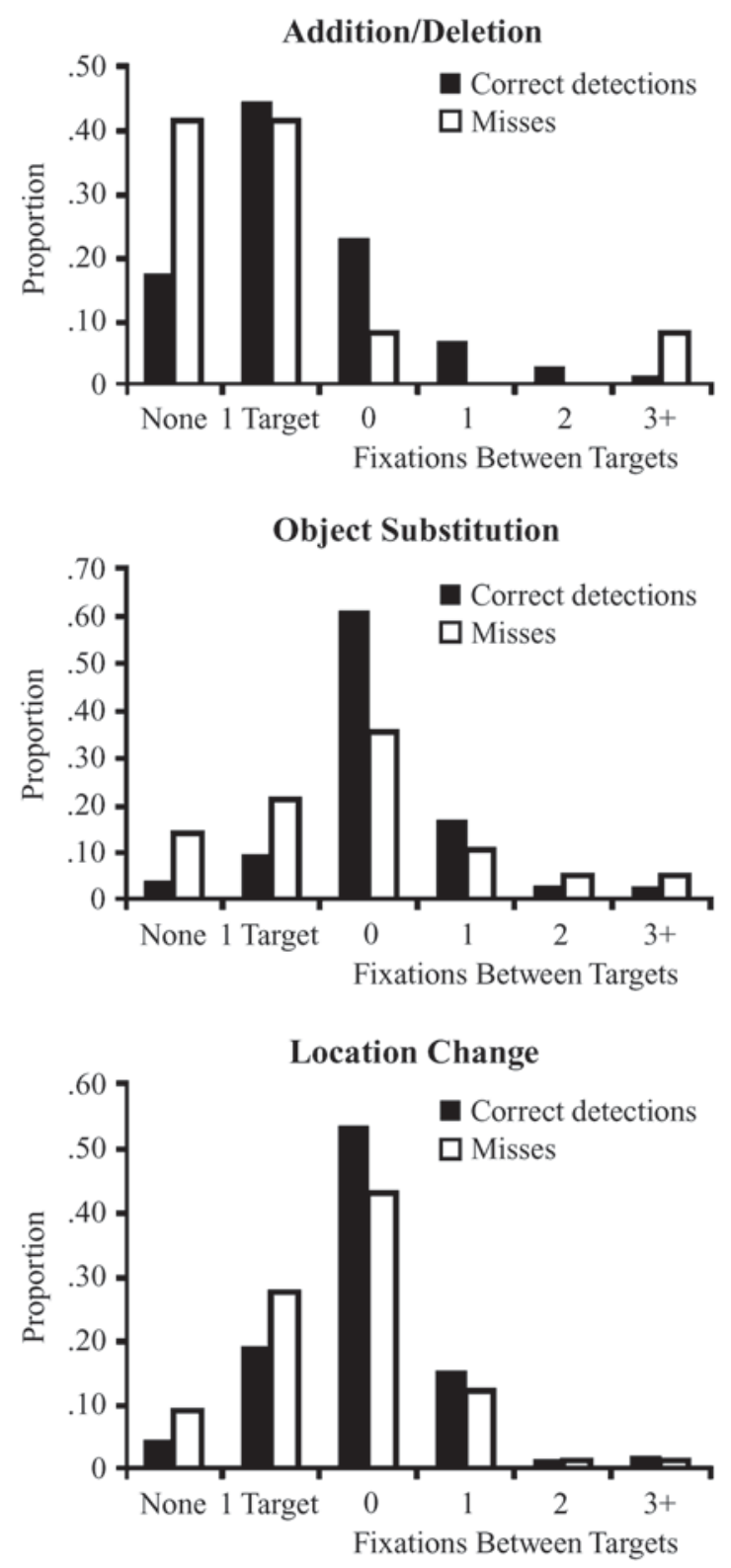

Figure 3. Target fixation behavior in Experiment 1. The chart shows the proportion of all trials in which the difference was detected (black bars) and in which the difference was missed (white bars); in which no targets were fixated (first category) or only one of the targets was fixated (second category); or if both targets were fixated, how many nontargets fell between consecutive views (the four rightmost categories).

Time course. To assess the time course of oculomotor variables, averages of three different measures were taken for each quartile of each trial. These measures were fixation duration, the proportion of saccades that switched between images (operationally defined along with presentation of the analysis), and the distance moved from corresponding points. Quartiles were calculated by dividing the number of eye movement events by four (for fixation 


\section{A) Number of Fixations Before First Target Fixation}

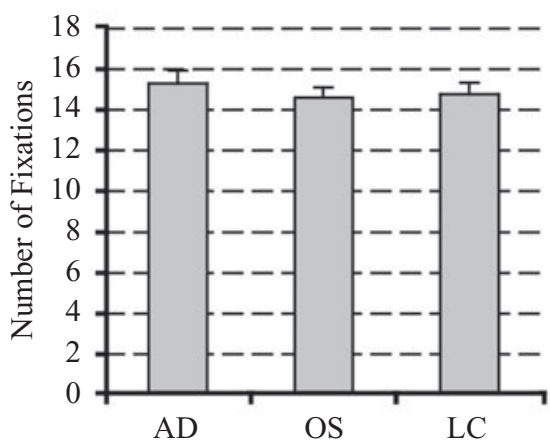

B) Distance Moved to First Target Fixation

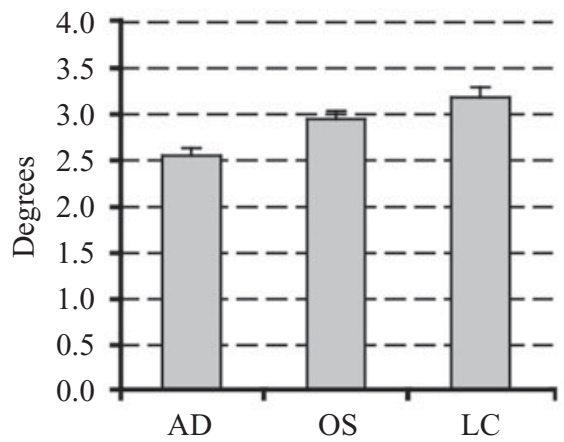

Figure 4. Peripheral guidance measures in Experiment 1. The charts show (A) the mean number of fixations before the first target fixation and (B) the distance moved when gaze was taken to the first target fixation. In both cases, the error bars represent the standard error of the mean. AD, addition/deletion; OS, object substitution; LC, location change.

duration, the number of fixations was used to calculate quartile size, and for the switching saccades and the distance moved from corresponding points, the number of saccades was used). For trials in which the total number of the appropriate measure was not divisible by four, eye movement events that lay on the cusp between two quartiles were included in both, ensuring an equal number of data points in each quartile. An example scan divided into four time quartiles can be seen in Figure 5. For each measure, means were taken for each participant for each of the three conditions and were analyzed using a 3 (difference type: $\mathrm{AD}, \mathrm{OS}$, or $\mathrm{LC}) \times 4$ (quartile: first, second, third, or fourth) repeated measures design. Figure 6 depicts the means for each of these measures for each difference condition at each quartile.

Fixation duration. The data were reciprocal transformed to ensure homogeneity of variance and to account for positive skew. A significant effect of quartile was found $[F(3,84)=31.051, p<.0001]$, but no main effects of difference type $(p>.1)$ or an interaction between them $(p>.1)$. The main effect of quartile resulted from significant differences between all the quartiles except the second and the third. Notably, differences existed between Quartiles 1 and 2 (198 and $209 \mathrm{msec}$, respectively; $q=$ $3.88, p<.05)$ and Quartiles 3 and $4(212$ and $230 \mathrm{msec}$, respectively; $q=7.56, p<.001$ ), due to longer fixations being made at the later quartiles.

Distance moved from corresponding points. To assess whether saccades were increasingly directed within corresponding regions of each image, saccade size was calculated as if there was only one image (as with the peripheral guidance analysis, above). This measure assessed the extent to which the participants refixated the same region and how far saccades took attention to a new search region. The means for each participant for each condition were reciprocal transformed (to adjust for substantial positive skew).
Again, a main effect of quartile was found $[F(3,84)=$ $341.134, p<.0001]$, due to significant differences between all quartiles, notably the first and the second $\left(3.5^{\circ}\right.$ and $2.2^{\circ}$, respectively; $q=29.52, p<.001$ ), the second and the third $\left(2.2^{\circ}\right.$ and $2.0^{\circ}$, respectively; $q=4.84, p<$ $.01)$, and the third and the fourth $\left(2.0^{\circ}\right.$ and $1.9^{\circ}$, respectively; $q=7.96, p<.001)$. This reduction in distance moved as the trial progressed demonstrates search becoming increasingly focused over time. A main effect of difference type was also obtained $[F(2,56)=15.986, p<$ $.0001]$, as was a significant interaction between difference type and quartile $[F(6,168)=9.263, p<.0001]$. The effect of difference was due to less focused search for $\mathrm{AD}$ than for OS $\left(2.5^{\circ}\right.$ and $2.3^{\circ}$, respectively; $q=7.81, p<$ $.001)$ and for LC than for OS $\left(2.5^{\circ}\right.$ and $2.3^{\circ}$, respectively; $q=5.40, p<.01)$. These differences seem to be due to the extent to which search becomes focused at the end of a trial: At the fourth quartile, OS search $\left(1.7^{\circ}\right)$ was more focused than was $\mathrm{AD}\left(1.9^{\circ} ; q=9.65, p<.001\right)$ and $\mathrm{LC}$ $\left(2.0^{\circ} ; q=9.90, p<.001\right)$ search.

Proportion of switches. The proportion of saccades that involved moving from one image to the other (switches) was calculated for each quartile. A saccade was defined as a switch if it began from farther right than the leftmost point of the right image and ended farther left than the rightmost point of the left image, or vice versa. A switching saccade is elicited when a comparison is about to be made, and thus the proportion of such saccades indicates how many eye movements are implicated in encoding before a comparison is elicited.

As before, a significant main effect of quartile was obtained $[F(3,84)=113.918, p<.0001]$, due to fewer switching saccades at the first than at the second quartile (.29 and .40 , respectively; $q=12.43, p<.001)$ and fewer at the third than at the fourth quartile (.43 and .52, respectively; $q=10.98, p<.001)$. No effect of difference type was found $(p>.1)$. However, a significant interac- 


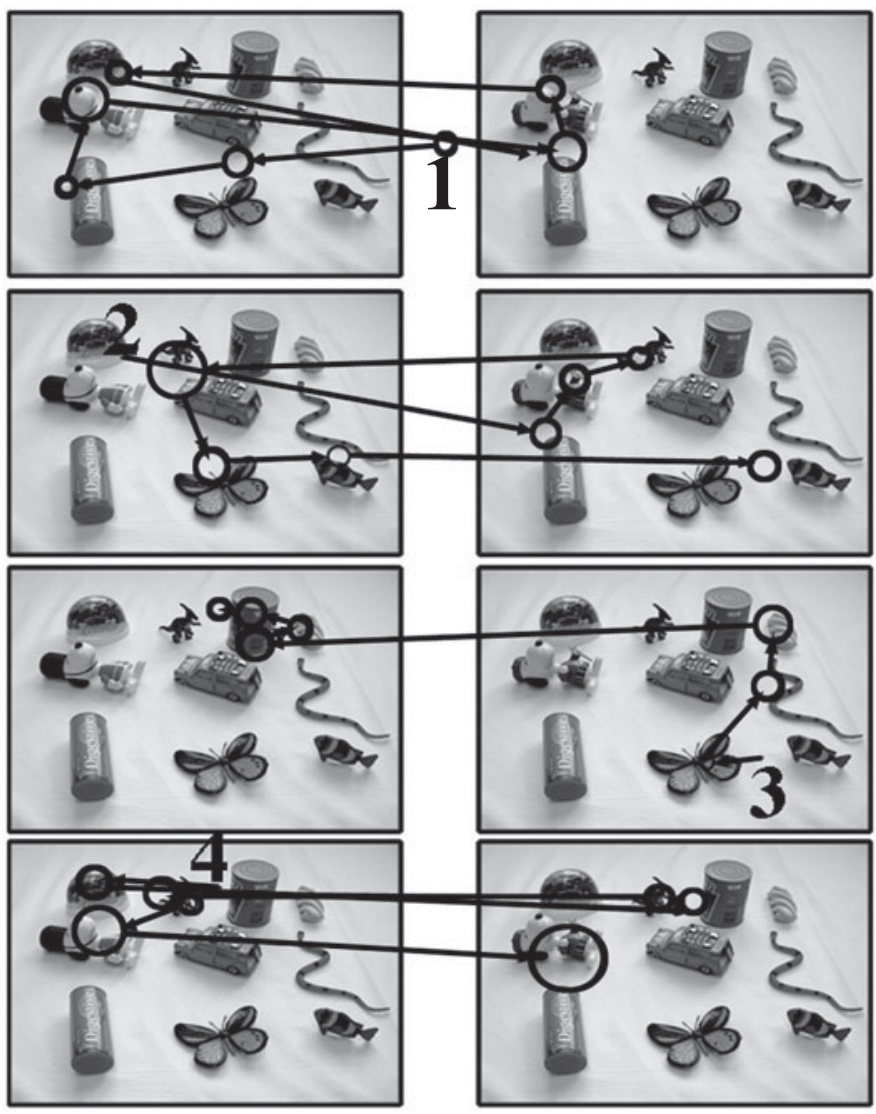

Figure 5. Scan path from Experiment 1 divided into four quartiles. The numbers indicate the start in each quartile. Circles represent fixations, and circle size corresponds to fixation duration. Lines represent saccadic eye movements.

tion was obtained between quartile and difference type $[F(6,168)=3.882, p<.01]$, due to differences between the $\mathrm{OS}$ and the $\mathrm{LC}$ conditions at the third quartile (.40 and .44 , respectively; $q=3.94, p<.05)$ and between $\mathrm{AD}$ and $\mathrm{LC}$ at the fourth quartile (.54 and .51 , respectively; $q=$ $3.54, p<.05)$.

\section{Discussion}

The results of Experiment 1 provide an initial indication of the way that search and comparison processes are coordinated in the CVS memory task. The robust time course effects suggest that, as the trial progresses, participants are inclined to narrow the focus of their search, fixate objects longer, and make more frequent comparisons. Similar findings have been reported in the literature before and have been summarized by the general rule "If you can't find what you're looking for, look more carefully" (Scinto et al., 1986, p. 290). However, although this trend was present throughout the whole trial for the distance-moved measure, a different story emerges for fixation duration and the proportion of comparative saccades. For these measures, the trends stop between the second and the third quartiles; instead, rises are apparent only from the first to the second quartile and from the third to the fourth quartile. A look at the scans indicates that eye movements during the first quartile are implicated in positioning the eyes at a good starting point, so it would seem that search takes place mainly during the second and third quartiles, whereas detection occurs during the fourth quartile. Therefore, the time course trends for fixation duration and the proportion of switching saccades are consistent with the suggestion of Pomplun, Sichelschmidt, et al. (2001) that different eye movement behavior is characteristic of detection. The aim of Experiment 2 was to shed further light on this issue by assessing eye movement behavior against a baseline consisting of matched same trials. If oculomotor variables were adjusted during the fourth quartile for different trials only, this would provide strong support for the detection hypothesis.

A further change was made to the stimuli in order to assess whether detection and verification processes are equivalent for the various difference types. In the time course analysis in Experiment 1, the focusing of search (as indicated by the distance-moved measure) was narrowed for the OS condition, relative to AD and LC. Although this is consistent with the difficulty of this condition relative 


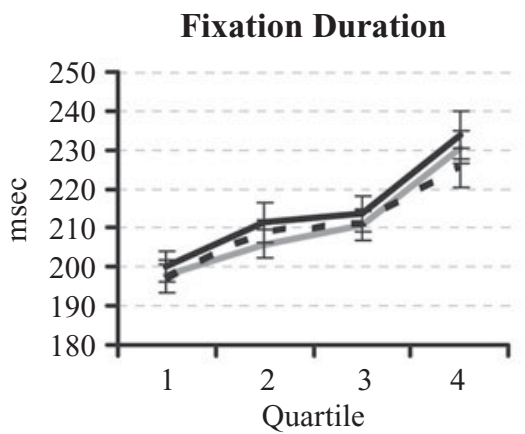

\section{Distance Moved From Corresponding Points}

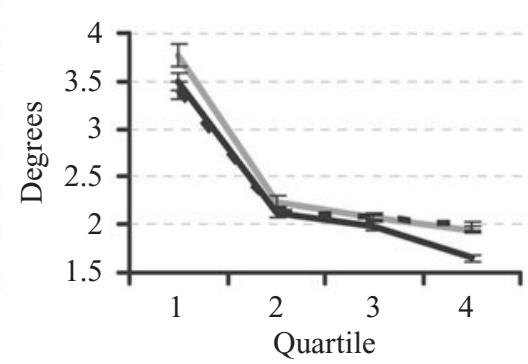

Switching Saccades

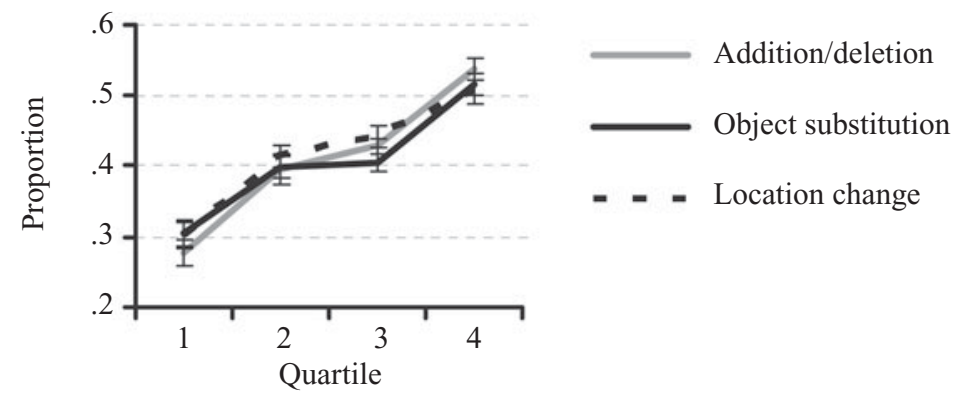

Figure 6. Charts depicting time course measures from Experiment 1, showing the means $( \pm S E)$ for addition/deletion, object substitution, and location change in each quartile.

to the $\mathrm{AD}$ condition, a simpler explanation is that the observers did not wish to fixate the blank regions of space created by the differences in the AD and LC conditions and, therefore, did not fixate the same locations back and forth during the verification process. Therefore, in Experiment 2, object flips about the vertical axis (which do not necessitate a change in spatial location) were introduced, instead of location changes. These act as a direct comparison with the OS condition and are assumed to be harder to discriminate.

A second feature of Experiment 1 concerned the lack of evidence for any peripheral detection of the differences. This was based on finding no advantage for the $\mathrm{AD}$ condition in terms of the number of fixations before the first target fixation or the distance moved toward the first target fixation (which ranged from $2.6^{\circ}$ to $3.2^{\circ}$ across conditions). However, Parker's (1978) evidence of peripheral detection was found when different trials were compared with same trials. Therefore, a further function of Experiment 2 was to reassess this peripheral guidance result against a same baseline. Furthermore, it is possible that the cluttered nature of the photographic stimuli used in Experiment 1 made differences harder to detect without direct fixation, and we were also concerned that the objects were not salient enough against their background. It was therefore decided to design a whole new set of stimuli consisting of spatially separated objects arranged on a white background. If peripheral guidance was possible, there would be a greater chance of observing it under these conditions.

Finally, the baseline same trials also allow further insight into why misses might occur, because they show search processes unaffected by detection and confirmation processes. Experiment 1 suggested that the observers missed the differences because (1) no target fixations occurred, (2) only one target was fixated, or (3) the two targets were not fixated consecutively. However, it is possible that the consecutive target fixations occurred after the difference had been detected and were indicative of confirmation, rather than search. In other words, these results might show the effect of detection on fixation, rather than the effect of fixations on detection. If this were the case, one would expect fixation behavior in the miss trials to be equivalent to that in the same trials, because in neither case would confirmatory fixations be expected. However, should fewer direct and consecutive fixations occur in the miss trials than in the same trials, it could be concluded that misses occur because of subnormal fixations allocated to the targets during search.

\section{EXPERIMENT 2}

\section{Method}

Participants. Twenty-eight members of the staff and student population ( 15 of them female) participated in return for $£ 4$. Four participants were replaced because they failed to detect any differences in 
the object flip condition. All the participants had normal or correctedto-normal vision, and none had taken part in Experiment 1.

Stimuli. The stimuli again consisted of paired pictures of children's toys. This time, each image contained nine individual photographs of toys from a bank of 152 toys, randomly assigned to 16 possible locations on a white background. At the viewing distance of $70 \mathrm{~cm}$, the images subtended $12.5^{\circ} \times 21.5^{\circ}$ and were separated by a blank region of $4^{\circ}$. Each half image contained one target object and eight distractors. The target objects differed in three ways: The difference could be the presence or absence of an object (AD; 16 items), the substitution of a different object (OS; 16 items), or an object flip about the vertical axis (OF; 16 items). Examples of a stimulus and the types of differences can be seen in Figure 7.

In addition, 16 filler items were included in which the difference was a size, rotation, or color change, in order to disguise the types of difference that might occur. Each image in which a difference was present (different trial) was matched with an identical image with no difference present (same trial), identical in every way except that, now, the same target object appeared in both image halves. The purpose of the same trials was to provide a baseline for eye movement behavior independent of detection.

Apparatus. The images were displayed on a computer monitor as described in Experiment 1. The eye-tracking details also replicated those in Experiment 1.

Design. The 128 trials (64 different trials +64 same trials) were rotated into two versions, such that no participant viewed a different trial and its matched same trial. The design was repeated measures, with 2 (trial type: different or same) $\times 3$ (difference type: $\mathrm{AD}, \mathrm{OS}$, or OF) factors (although the same trials did not vary in terms of difference type, they were treated as such in order to isolate any extraneous variables, such as image complexity, that might unintentionally differ between conditions).
Procedure. The procedural details follow those in Experiment 1. The only difference was that feedback was not provided upon response.

\section{Results}

The 4 participants who exhibited low accuracy and failed to detect any differences in the OF condition were removed from all the analyses except the accuracy analysis. The filler trials were also removed from analysis. The remaining data were analyzed for accuracy, reaction time, and number of fixations, using a 2 (trial type: different or same) $\times 3$ (difference type: AD, OS, or OF) repeated measures ANOVA. Figure 8 shows means for each measure for each condition. Significant main effects were found for difference type in accuracy $[F(2,62)=42.532, p<.0001]$, reaction time $[F(2,54)=19.394, p<.0001]$, and number of fixations $[F(2,54)=18.614, p<.0001]$. Furthermore, difference type interacted with trial type [accuracy, $F(2,62)=$ $39.189, p<0001$; reaction time, $F(2,54)=15.429, p<$ .0001 ; number of fixations, $F(2,54)=19.72, p<.0001]$. The interaction in all cases was due to an effect of difference type for different trials and no effect of difference type for same trials, demonstrating that the three difference type conditions varied by difference type only. The effect of difference type was due to a speed and accuracy advantage for deletions over substitutions and for substitutions over flips. Responses to AD were more accurate than those to OS (94\% vs. $82 \% ; q=3.66, p<.05)$, which

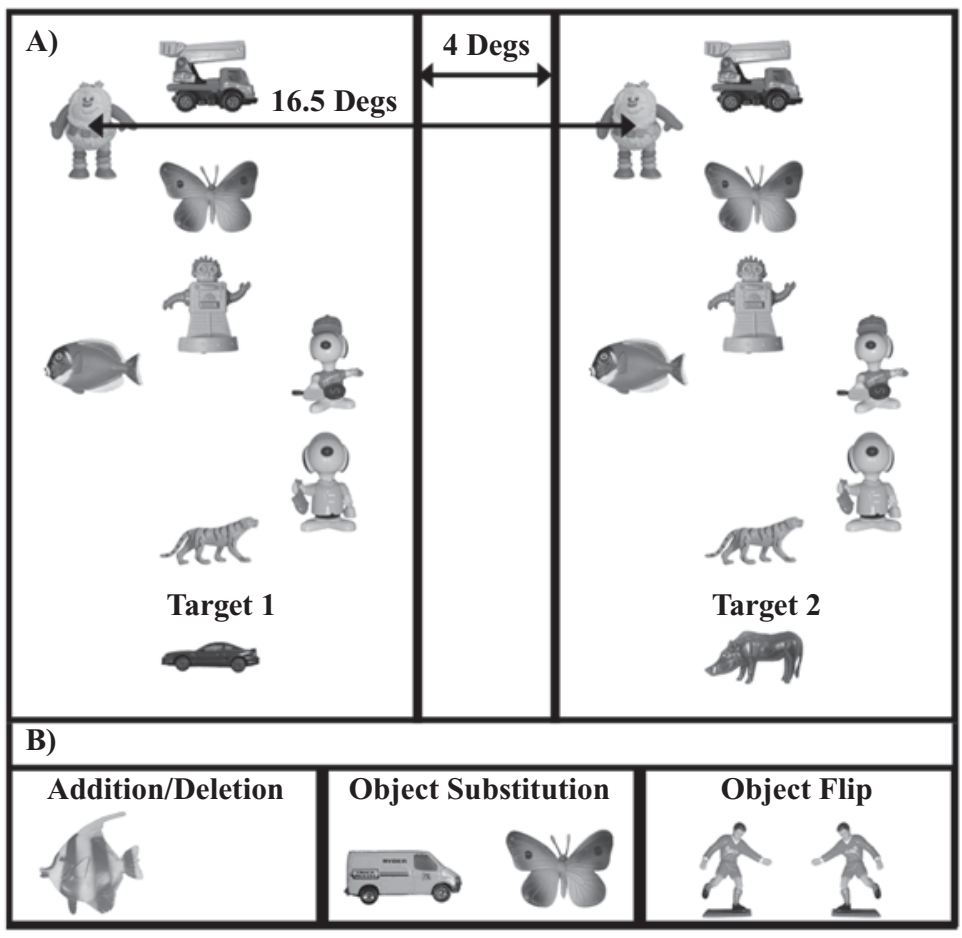

Figure 7. Stimuli from Experiment 2. (A) Example of stimuli from the object substitution condition. (B) Example target objects from each difference type condition. 
A) Accuracy

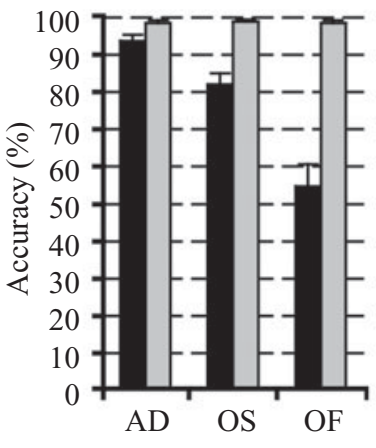

C) Number of Fixations

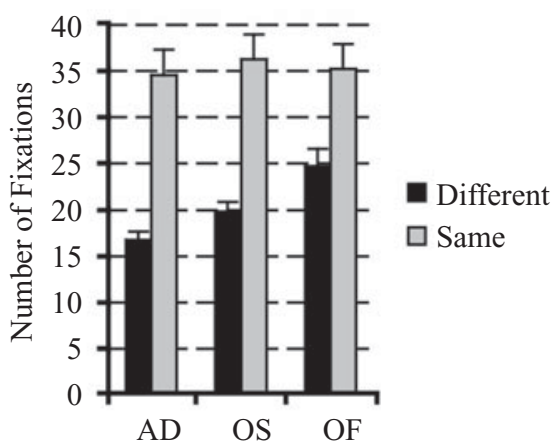

B) Reaction Time

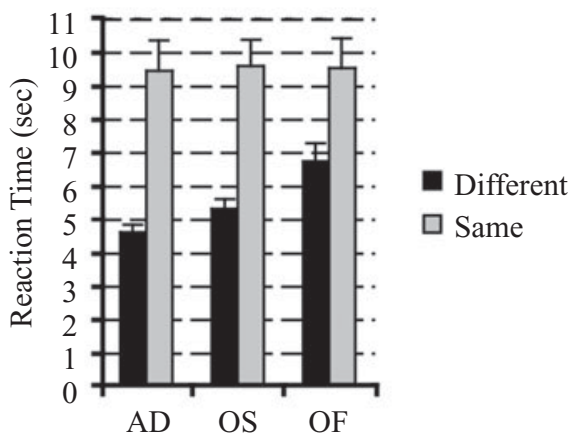

D) Number of Target Fixations

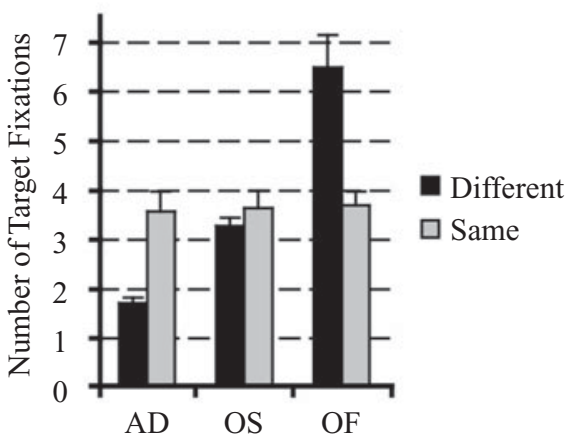

Figure 8. Measures of performance from Experiment 2, showing the mean and standard error of the mean for each condition. AD, addition/deletion; OS, object substitution; $O F$, object flip.

in turn were more accurate than those to $\mathrm{OF}(55 \% ; q=$ $9.02, p<.001)$. Responses to $\mathrm{AD}$ were also faster than those to OS (4.6 vs. $5.3 \mathrm{sec} ; q=5.11, p<.01$ ), which in turn were faster than those to OF $(6.8 \mathrm{sec} ; q=3.65$, $p<.05)$. Finally, in line with reaction time, responses to AD needed fewer fixations than did those to OS (16.8 vs. 19.8; $q=5, p<.01)$, which in turn needed fewer fixations than did those to OF $(24.7 ; q=3.59, p<.05)$. The difference type manipulation was, therefore, successful in creating two types of difference (OS and OF) that varied with ease of detection without altering the spatial layout of the displays. An example scan from Experiment 2 can be seen in Figure 9.

Target fixations. As in Experiment 1, an analysis was conducted on the number of target fixations, this time including baseline same trials (see Figure 8D). A main effect of difference type was obtained $[F(2,54)=46.525$, $p<.0001]$, which interacted with trial type $[F(2,54)=$ $35.428, p<.0001]$. As with the previous analyses, the interaction was due to an effect of difference type for the difference trials only. Specifically, the AD condition required fewer target fixations (1.7) than did the OS condition (3.3; $q=4.48, p<.01$ ), which in turn required fewer fixations than did the OF condition $(6.5 ; q=8.92, p<.001)$.

In terms of target fixations and detection, a more consistent pattern was observed than in Experiment 1. Fewer target-directed fixations occurred on miss trials than on correct trials in the AD condition (1.2 and 1.7, respectively), the OS condition (2.5 and 3.3, respectively), and the OF condition ( 3.1 and 6.5 , respectively). However, this still does not determine whether detection precedes or follows direct fixation; thus, a more fine-grained analysis of the target fixations was conducted. Figure 10 shows the outcome of this analysis, in which the black bars show the proportions of all correct detections, the gray bars show the proportions of correct rejections in the same trials (normal target fixation behavior in the absence of detection), and the white bars show the proportions of misses. Turning first to the correct detections, it is clear that most trials were characterized by direct and consecutive fixations on both targets, at least in the OS and OF conditions (.66 and .8 respectively). Interestingly, the proportion of correct detections with no target fixations was 0 in the OF condition and less than .1 in the OS condition, showing the strength of the tendency to fixate the targets. It is also clear that some of these target fixations occurred after detection, as comparison with the same trials shows: Fewer consecutive target fixations occurred in the same trials in the OS condition (.55) and the OF condition (.62). However, it is also clear that the miss trials exhibited less systematic search behavior than did the same trials. In the miss trials, consecutive inspection of the targets accounts for only .22 of the trials in the OS condition and . 39 in the OF condition. Furthermore, more miss trials were charac- 


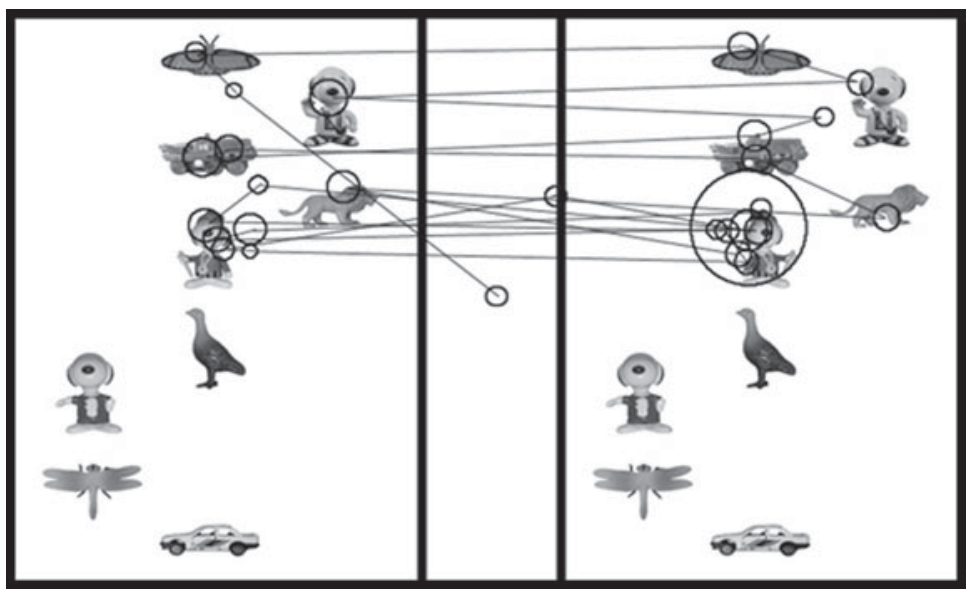

Figure 9. Example scan from Experiment 2 depicting a typical search, which moved first to the top of the display before rapidly scanning each object in turn. Upon reaching the targets (flipped Snoopy toy), long fixations and multiple switching saccades were employed.

terized by the fixation of only one target. The consistent pattern observed across the OS and OF conditions suggests that (1) misses occurred because the targets were not consecutively fixated, (2) consecutive target fixations were common to search in the absence of detection, and (3) further consecutive fixations were more likely to occur after detection. Considering the $\mathrm{AD}$ condition is again problematic, due to the existence of only one real target in this condition. Nevertheless, it would appear that some errors occurred because only one target was fixated.

Peripheral guidance. Analyses of the number of fixations before the target was fixated and the distance moved into the target area were conducted using a trial type $X$ difference type ANOVA. The data are shown in Figure 11. As with Experiment 1, no effect of the number of fixations was observed for difference type $(F<1)$. Furthermore, targets were not fixated earlier in the different trials $(F<$ $1)$, and no interaction was obtained $(F<1)$. Therefore, even though the targets were highly visible against a blank background, there was no advantage over the same trials. The distance-moved measure did obtain a significant effect of difference type $[F(2,54)=7.499, p<.01]$, and this was due to a greater distance moved in the OS condition $\left(5^{\circ}\right)$ than in the $\mathrm{AD}$ condition $\left(4.5^{\circ} ; q=4.17, p<.05\right)$ and the OF condition $\left(4.4^{\circ} ; q=5.16, p<.001\right)$. However, there was no effect of trial type $(F<1)$ or any interaction $(F<1)$, suggesting that the effect in the OS condition may have been due to an irregularity in the spatial layout of items in this condition (OS same trials, 4.9 ; OS different trials, $5.1^{\circ}$ ). As with Experiment 1, very little evidence was obtained of peripheral guidance of gaze toward the target items.

Time course. The purpose of this experiment was to compare search behavior for different trials with that for same trials in order to isolate the effects of detection on the time course trends observed in Experiment 1. The crucial comparisons therefore concern what occurred at the end of trials as measured in the fourth quartile. Charts of each time course measure for each difference type condition and an average of the same trials (collapsed across the three conditions for clarity) are shown in Figure 12.

Fixation duration. For the fixation duration analysis, the data were reciprocal transformed in order to reduce positive skew. The effect of quartile for fixation duration was replicated $[F(3,81)=48.076, p<.0001]$, with longer fixations at the second quartile $(203 \mathrm{msec})$ than at the first quartile $(191 \mathrm{msec} ; q=7.65, p<.001)$ and at the fourth quartile $(218 \mathrm{msec})$ than at the third quartile (205 msec; $q=7.30, p<.001)$. No effect of trial type was obtained $(p>.1)$, indicating that fixations were not greater when a difference was present. However, a significant trial type $\times$ quartile interaction $[F(3,81)=8.569$, $p<.0001]$ was observed, due to longer fixations during the last quartile when a difference was present [224 vs. $212 \mathrm{msec} ; F(1,27)=4.518, p<.05]$. When same trials were considered separately, no significant rise in fixation duration occurred after the second quartile, suggesting that the lengthy searches did not encourage an adjustment in looking time.

Distance moved from corresponding points. For the analysis considering the distance moved from corresponding points, a slightly different pattern was observed from that seen in Experiment 1. A significant effect of quartile $[F(3,81)=107.223, p<.001]$ was due only to less focused saccades at the beginning of a trial (first quartile, $\left.4.2^{\circ}\right)$. No differences existed between the second $\left(3.1^{\circ}\right)$, third $\left(3.1^{\circ}\right)$, and fourth $\left(3.1^{\circ}\right)$ quartiles. Furthermore, saccades were less focused during the first quartile for trials containing a difference $\left[4.6^{\circ}\right.$ vs. $3.9^{\circ} ; F(1,27)=45.682$, $p<.0001]$. It is hard to see how the presence of a difference could exert an effect at the beginning of a trial; thus, this result is likely to have been due to the fact that there were more fixations in the same trials. This means that more saccades contributed to the averages derived 

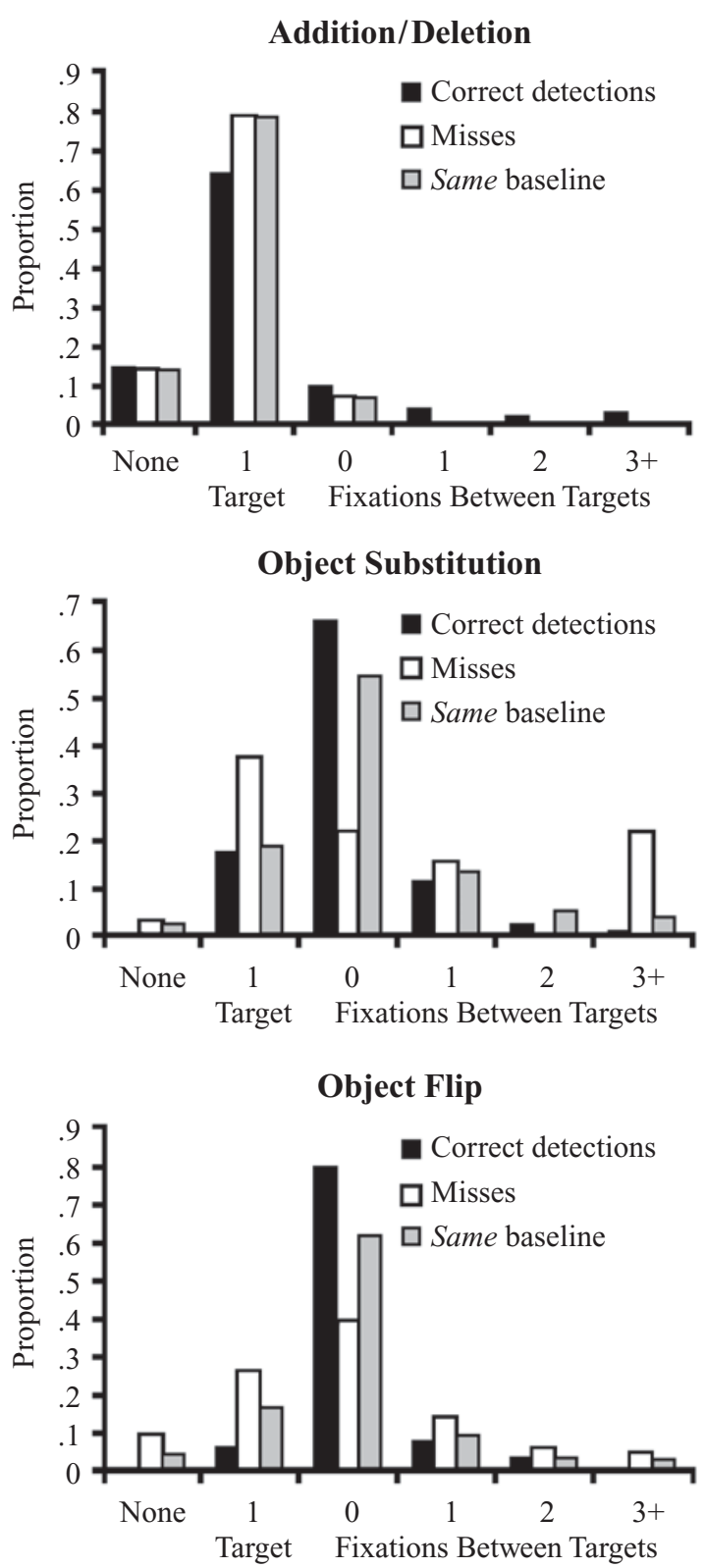

Figure 10. Target detection behavior in Experiment 2. The charts show the proportions of all trials on which the difference was detected (black bars) and on which the difference was missed (white bars), as well as the proportions in the baseline same trials (gray bars).

for each quartile in the same trials, diluting the influence of the first few eye movement events implicated in positioning gaze. (Since this issue might also be relevant to the detection phase, an absolute analysis of the last five eye movements is included, below.) However, this issue cannot explain why saccades in the OF condition were significantly more focused than those in the OS condition in the third quartile $\left(2.9^{\circ}\right.$ vs. $\left.3.3^{\circ} ; q=4.37, p<.01\right)$ and, in the fourth quartile, were more focused than those in both the OS $\left(2.3^{\circ}\right.$ vs. $\left.3.2^{\circ} ; q=6.21, p<.001\right)$ and the
$\mathrm{AD}\left(3.5^{\circ} ; q=6.88, p<.001\right)$ conditions. These differences can be due only to a substantial narrowing of the search focus upon detection for the difficult OF condition and are not an effect of the larger number of saccades in this condition, since that would serve to reduce any effect of detection in the same way as the same trials described above.

Proportion of switches. Finally, the same time course trend as that observed in Experiment 1 was obtained for the proportion of switching saccades $[F(3,81)=95.922$, $p<.0001$ ], due to less comparative saccades being made in the first (.29) than in the second (.40) quartile $(q=$ $15.84, p<.001)$ and in the third (.40) than in the fourth (.45) quartile $(q=7.26, p<.001)$. An interaction was observed between quartile and trial type $[F(3,81)=23.831$, $p<.0001$ ], due to a higher proportion of switching saccades at the fourth quartile when a difference was present [different $=.48$ vs. same $=.42 ; F(1,27)=38.111, p<$ $.0001]$. However, caution should be used before attributing this effect to detection, since an effect was also observed during the first quartile, due to more comparisons being made when no difference was present [different $=$ .25 vs. same $=.32 ; F(1,27)=50.571, p<.0001]$. What is clear, however, is that although both different and same trials exhibited an increase in comparative saccades from the first to the second quartile (same, $q=7.72, p<.001$; different, $q=14.69, p<.001$ ), only the different trials showed an increase from the third to the fourth quartile (same, $q=2.14, p>.05$; different, $q=8.12, p<.001$ ). This demonstrates that an increase in comparative saccades $i$ the result of detection.

Analysis of the last five eye movements. As was described above, one concern with the quartile analyses is that the conditions vary in terms of the number of fixations or saccades within a trial and, therefore, different numbers of eye movement events contribute to each quartile. This is a particular risk when trials containing a difference are compared with the same trials: A slight change in eye movement behavior at the end of search may have a significant impact when there are few fixations but may be hidden when one is looking at lengthy searches. To check that the time course trends were not an artifact of the quartile method, an analysis was conducted of the last five eye movement events leading up to response. Each difference condition was analyzed separately using a 2 (trial type: different or same) $\times 5$ (fixation before response [R]: R-5, R-4, . R R-1) ANOVA, and this was conducted for each time course measure (fixation duration, the distance moved from corresponding points, and the proportion of switches). It was important to exclude the very short fixations that characterized Quartile 1, and therefore only trials containing more than seven fixations were included. This did, however, mean that 2 participants provided no data in the OF condition, and thus the analysis for this difference type was based on 26 participants.

The results (shown in Figure 13 and Table 1) indicated that the detection and verification phase is limited to the last few saccades and fixations preceding response but, also, is (almost) unique to trials containing a difference. 
A) Number of Fixations Before First Target Fixation

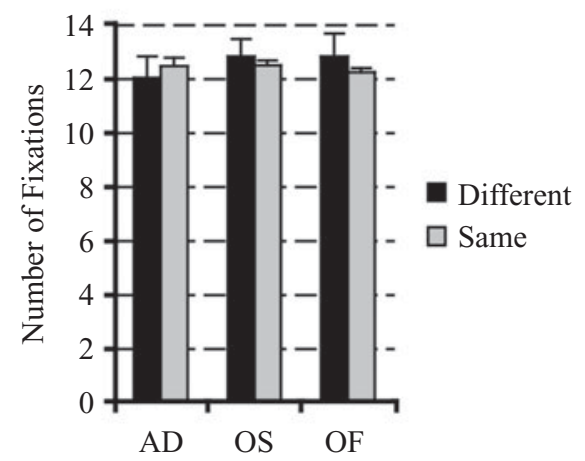

B) Distance Moved to First Target Fixation

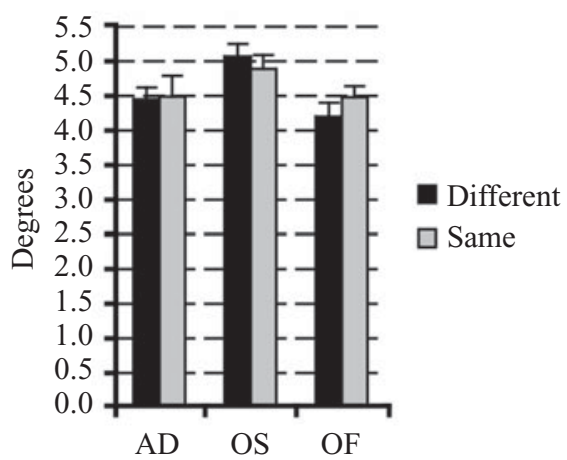

Figure 11. Peripheral guidance measures in Experiment 2. Error bars represent the standard error of the mean. AD, addition/deletion; OS, object substitution; OF, object flip.

Turning first to fixation duration, it was found that the point at which the fixation duration of different trials became greater than that of same trials corresponded to the difficulty of that condition, as revealed by RT and accuracy. For the easiest (AD) condition, no effects were observed $(F<1)$. However, a rise in fixation duration was observed at the last fixation in both same and different trials, meaning that an effect of fixation number was observed $[F(1,27)=11.164, p<.0001]$ which did not interact with trial type $(p>.49)$. However, this rise in fixation duration for same trials appears to be an anomaly and was not observed for either the OS or the OF condition. In the OS condition, a significant interaction between trial type and fixation number was obtained $[F(4,108)=4.651, p<$
Fixation Duration

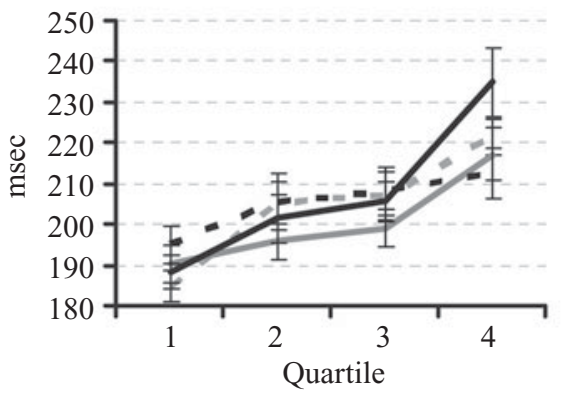

Switching Saccades

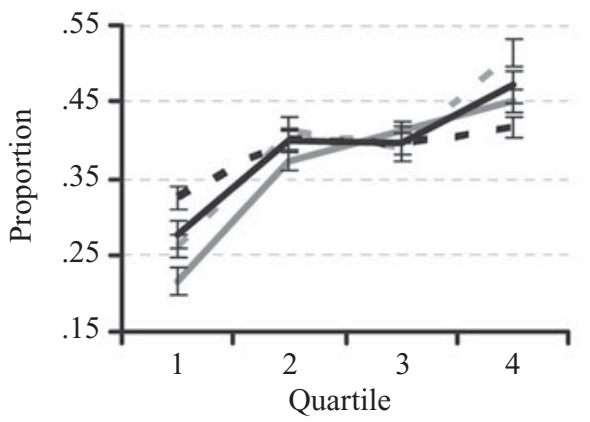

Distance Moved From Corresponding Points

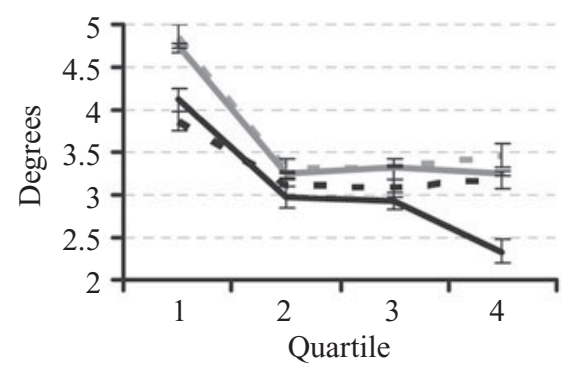

$=-=$ Addition/deletion

Object substitution

Object flip

- = Same baseline

Figure 12. Charts depicting time course measures in Experiment 2, showing the means $( \pm S E$ ) for addition/deletion, object substitution, and object flip at each quartile. In the interest of clarity, the baseline depicts the mean for all same conditions (which did not differ statistically) and the average standard error for these conditions. 

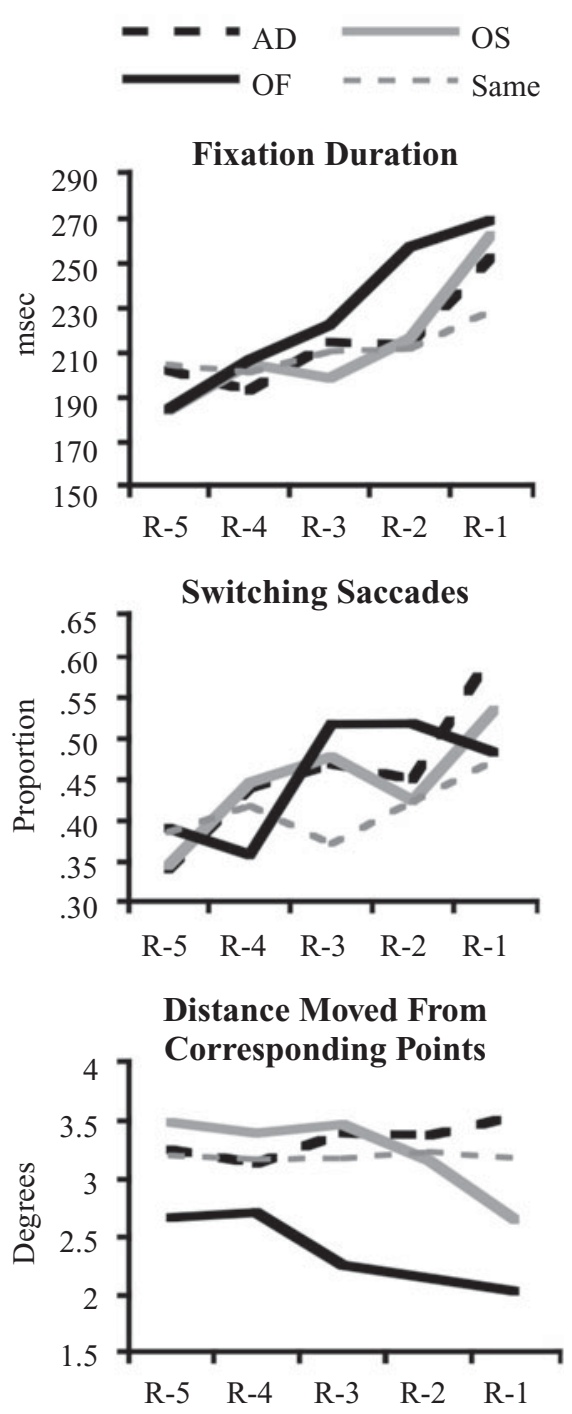

Figure 13. Mean durations of the last five fixations leading up to response in Experiment 2. In the interest of clarity, the baseline depicts the mean for all same conditions. AD, addition/deletion; OS, object substitution; OF, object flip. R, response.

.002], due to an increase in fixation duration at the last fixation $(\mathrm{R}-1 ; p<.001)$. For the hardest $(\mathrm{OF})$ condition, a significant interaction was also obtained $[F(4,100)=$ $5.159, p<.001]$, this time due to differences at R-1 ( $p<$ $.025)$ and $\mathrm{R}-2(p<.02)$. In neither condition was an effect in same trials observed $(p \mathrm{~s}>.05)$. These results confirm that increases in fixation duration coincide with detection and that the increases last longer, depending on the difficulty of the discrimination.

The results of fixation duration were mirrored in the analysis looking at the distance moved from corresponding points. In the $\mathrm{AD}$ condition, no main effects or interactions were obtained ( $p \mathrm{~s}>.1)$. For the OS condition, an interaction between trial type and saccade number $[F(4,108)=3.527, p<.01]$ was due to a reduction in the distance moved at the last saccade only $(p<.03)$. For the OF condition, the interaction $[F(4,100)=2.586, p<$ $.05]$ was due to a reduction in distance moved that had diverged significantly from that in the same trials by R-3 $(p<.01)$ and was maintained through $\mathrm{R}-2(p<.01)$ and $\mathrm{R}-1(p<.01)$. It appears that both an increase in fixation duration and a reduction in the area under scrutiny are characteristic of detection for the OSs and OFs, whereas little evidence can be found for an independent detection phase in the easy AD condition. However, the proportion of saccades that switched between images was found to be sensitive to detection in this condition. An interaction between trial type and fixation number $[F(4,108)=$ $2.678, p<.05]$ was due to an increase in the proportion of switching saccades during $\mathrm{R}-1$ only $(p<.01)$. In contrast, no effects were observed in the OS condition, suggesting that detection is not served by an increase in comparative saccades in this condition. For the OF condition, an interaction was obtained $[F(4,100)=3.386, p<.05]$, and this was due to an increase in comparative saccades at R-3 $(p<.001)$ and $\mathrm{R}-2(p<.05)$, although this increase was not evident during the last saccade at R-1 $(p>.4)$.

Overruns. One last finding of interest concerns the number of instances in which the targets were fixated, left, and then immediately returned to, suggesting that the contents of the previous fixation had not been fully processed before the decision to move the eyes had been executed. To assess the number of such cases, an operational definition was formulated in which both targets must be consecutively fixated, gaze must be directed to another object, and gaze must then return to the target within three fixations. This happened in $4.6 \%$ of the correct detections in the $\mathrm{AD}$ condition, $12.1 \%$ in the $\mathrm{OS}$ condition, and $23.4 \%$ in the OF condition. Such a finding is interesting, since it suggests that detection is not served by a systematic comparison of target elements but, rather, is the result of rapid scanning in which the difference pops out.

\section{Discussion}

The general pattern of results observed in Experiment 2 is in agreement with that seen in Experiment 1. Again, null results were obtained for the peripheral guidance analyses, suggesting that attention is focused narrowly during search. The error analysis indicated that objects are generally consecutively fixated in the absence of detection, concurring with the peripheral guidance results and suggesting that an object-by-object comparison strategy characterizes search.

In terms of time course, fixation duration rises from the start of the trial, reaches a plateau for a lengthy period during the middle portion of a trial, and then rises again shortly before response. In addition to the trend shown in the previous experiment, the present data confirm that this rise is limited to trials containing a difference and, thus, can be attributed to detection and verification. The same pattern is observed in the proportion of saccades implicated in comparisons. The trend for the distance-moved measure was not replicated from Experiment 1. Apart 
Table 1

Fixations Before Response (R)

\begin{tabular}{|c|c|c|c|c|c|c|c|c|c|c|}
\hline & \multicolumn{2}{|c|}{$\mathrm{R}-5$} & \multicolumn{2}{|c|}{$\mathrm{R}-4$} & \multicolumn{2}{|c|}{$\mathrm{R}-3$} & \multicolumn{2}{|c|}{$\mathrm{R}-2$} & \multicolumn{2}{|c|}{$\mathrm{R}-1$} \\
\hline & $M$ & $S D$ & $M$ & $S D$ & $M$ & $S D$ & $M$ & $S D$ & $M$ & $S D$ \\
\hline \multicolumn{11}{|c|}{ Fixation Duration (msec) } \\
\hline AD diff & 202 & 38 & 193 & 27 & 215 & 45 & 214 & 37 & 253 & 60 \\
\hline AD same & 203 & 29 & 206 & 41 & 212 & 39 & 212 & 44 & 242 & 66 \\
\hline OS diff & 184 & 28 & 205 & 26 & 199 & 31 & 217 & 37 & 262 & 75 \\
\hline OS same & 200 & 35 & 198 & 29 & 204 & 40 & 212 & 36 & 221 & 41 \\
\hline OF diff & 185 & 39 & 207 & 46 & 223 & 51 & 257 & 108 & 269 & 74 \\
\hline OF same & 212 & 42 & 200 & 38 & 217 & 48 & 212 & 34 & 223 & 62 \\
\hline \multicolumn{11}{|c|}{ Distance Moved (deg) } \\
\hline AD diff & 3.25 & 1.15 & 3.14 & 0.83 & 3.40 & 1.23 & 3.38 & 0.72 & 3.54 & 1.00 \\
\hline AD same & 3.22 & 1.08 & 3.08 & 1.11 & 2.99 & 0.92 & 3.26 & 1.18 & 2.99 & 0.97 \\
\hline OS diff & 3.49 & 1.20 & 3.40 & 1.05 & 3.47 & 1.57 & 3.16 & 1.19 & 2.65 & 1.14 \\
\hline OS same & 3.17 & 0.95 & 3.57 & 0.96 & 3.23 & 1.21 & 3.22 & 1.16 & 3.47 & 1.01 \\
\hline OF diff & 2.67 & 0.79 & 2.71 & 1.13 & 2.26 & 1.05 & 2.15 & 0.85 & 2.03 & 1.00 \\
\hline OF same & 3.22 & 1.00 & 2.86 & 0.67 & 3.32 & 0.93 & 3.23 & 1.07 & 3.07 & 1.21 \\
\hline \multicolumn{11}{|c|}{ Proportion of Switches } \\
\hline AD diff & .34 & .20 & .44 & .21 & .47 & .20 & .45 & .27 & .61 & .20 \\
\hline AD same & .39 & .20 & .40 & .20 & .41 & .18 & .45 & .18 & .43 & .19 \\
\hline OS diff & .35 & .19 & .45 & .21 & .48 & .22 & .42 & .21 & .54 & .17 \\
\hline OS same & .41 & .21 & .40 & .15 & .40 & .17 & .43 & .18 & .46 & .25 \\
\hline OF diff & .39 & .28 & .36 & .31 & .52 & .25 & .52 & .23 & .48 & .29 \\
\hline OF same & .36 & .16 & .45 & .19 & .31 & .16 & .39 & .18 & .52 & .18 \\
\hline
\end{tabular}

from a decrease in the focus of the search from the first to the second quartile, no other adjustments over time were observed. The exception to this rule was the difficult OF condition, which showed a considerable narrowing of the search focus that began at the third quartile. The idea that the difficulty of the discrimination could influence the behavior observed during detection and verification was confirmed by the onset of the increase in fixation duration and the decrease in distance moved, which corresponded directly with the ease with which the difference types could be detected.

\section{GENERAL DISCUSSION}

The experiments reported here were designed to assess several aspects of eye movement behavior during a memory task in which encoding and comparison constraints were removed. We wondered about the strategies employed, given that previous visual memory tasks had observed strategic adaptations to stimulus presentation changes (see, e.g., Melcher \& Kowler, 2001; Nelson \& Loftus, 1980). As our starting point, a hypothesis was tested, that the oculomotor behavior implicated in detection and verification processes by Pomplun, Sichelschmidt, et al. (2001) was, rather, the result of time course adjustments in scanning behavior observed in scene perception (Antes, 1974; Buswell, 1935; Irwin \& Zelinsky, 2002) and continuous search (Scinto et al., 1986). Two key findings from the present experiments provide strong support for the conclusions drawn by Pomplun, Sichelschmidt, et al.: (1) The adjustment in oculomotor variables was not continuous, with no major changes observed between the second and third quartiles, and (2) the changes that occurred between the third and fourth quartiles were observable only when a difference was present. Therefore, the present data concur with the suggestion that comparative visual search is characterized by a lengthy search and comparison phase (observed during the second and third quartiles) and a brief detection and verification phase (observed during the fourth quartile). The transition between the two is marked by an increase in fixation duration, an increase in comparative saccades directed back and forth, and in some cases (i.e., for difficult discriminations), a decrease in the size of the area under focal scrutiny. One possibility is that the behavior observed during detection is, instead, the result of observers' holding their gaze on a target while producing a manual response. ${ }^{1}$ However, the fact that detection behavior varied according to the type of discrimination argues against this possibility. Instead, it would seem that the comparative saccades, longer fixations, and narrowing of the search region play functional roles in detection. In addition to further establishing the different search phases, the present results also extend Pomplun's findings to scene-like images depicting real objects.

One finding observed here but not reported by Pomplun, Sichelschmidt, et al. (2001) concerns the change in eye movement behavior from the first quartile to the second. This robust trend was observed for each measure in both experiments. The characteristics of this phase (short fixations, directed far apart from each other) are consistent with suggestions in the scene perception literature of an initial orienting phase in which image features are rapidly scanned (Karpov, Luria, \& Yarbus, 1968), possibly to map salient features in order to plan a future scan path. However, a look at the locations of the initial few fixations 
in the present data suggests that their purpose may simply have been to position the eyes at a good starting point to conduct a systematic search that did not double back on itself (i.e., top to bottom). This could be checked easily by seeing whether the existence of the phase depends on the position of the initial fixation point.

Once the eyes had been taken to a suitable starting point, the search phase began. This seems to have been characterized by a rather rapid scan back and forth, with average fixation duration during the second and third quartiles reaching only around $210 \mathrm{msec}$ in Experiment 1 and $204 \mathrm{msec}$ in Experiment 2. This is considerably shorter than the average fixation duration normally observed in both visual search and scene perception (estimated to be around 275 and $330 \mathrm{msec}$, respectively; Rayner, 1998). The rapid nature of this scan is further highlighted by the instances in Experiment 2 in which the scan moved past the targets before promptly returning. Similar findings in the visual search literature (Gould, 1973; Hooge \& Erkelens, 1996) have been interpreted as demonstrating that full analysis of the current fixation is not always utilized for the programming of the next saccade (Hooge \& Erkelens, 1998). The implications of this finding for the present task are that participants are already looking ahead, beyond the output of the current comparison operation. Furthermore, the frequency of these instances across the different conditions pinpoints the time course with which object information becomes available for comparison: the presence of an object, the identity of that object, and the orientation of that object. If fixation duration is not expanded sufficiently to acquire the necessary information, the difference may be missed before the following eye movement is executed. The pattern of results from the error analyses is in agreement. In the cases in which both targets were consecutively fixated, more of these led to error in the hard OF condition (.39) than in the easier OS condition (.22). These instances perhaps resulted from the same phenomena as the overruns: Gaze had left the targets before the crucial information was acquired.

A further feature of the search phase is the high proportion of comparative saccades: .42 in Experiment 1 and .40 in Experiment 2. This means that only two or three fixations were directed within an image before a comparison was elicited, suggesting that the observers in this task preferred to actively scan the displays to cut down on memory usage. Of course, it is possible that multiple objects could be encoded within each of these fixations, but the peripheral guidance analyses argue against this option. If multiple objects were encoded without fixations on each, one would expect to find evidence that the targets were fixated sooner and were oriented to from a farther distance than were the nontargets. This was not the case, with the targets apparently not being detected until they were the next objects to be searched. The error analyses also support this interpretation. Both experiments produced evidence suggesting that misses occurred because the targets were not consecutively fixated, and Experiment 2 confirmed that consecutively fixating the targets was the most common way for search to proceed, even when there was no difference to be detected. The evidence supports an object-byobject comparison strategy in which only a single object is encoded from each image, whereas even the presence of surrounding objects is not compared across images.

A similar preference for active scanning over memory usage was reported by Ballard, Hayhoe, and Pelz (1995), in which eye movements were shown to be used to minimize the need for working memory in a block-copying task. Specifically, the participants used separate fixations to encode the color and location of a block, rather than employing a single fixation and loading visual memory with both features. It is worth noting that the displays in the block-copying task were static and remained ever present, as in comparative visual search. These findings suggest that when observers are free to view displays without timing constraints, their preference is to employ many brief fixations, to avoid burdening short-term memory. What implications might this have for tasks that employ paradigms with fixed timing constraints? Shore and Klein (2000) have suggested that search in spot-the-difference tasks is under endogenous control, whereas control is removed from the observer in flickering displays. Given that the peripheral detection observed by Parker (1978) and Zelinsky (2001) was observed under artificial viewing conditions, this may suggest that the selection of visual detail in more naturalistic tasks, when the observer has active control, is limited and does not utilize information beyond fixation.

The final phase occurs upon detection of a suspicious object, eliciting a series of deliberate and controlled eye movements between the potential targets. That the length of this phase varies with the type of difference suggests that detection (or at least detection with full confidence) is not an all-or-nothing event but, rather, a gradual process that takes time to resolve. The suggestion has already been made in the change detection literature to explain the finding that participants can sometimes specify that something has changed without identifying the nature of the change (see, e.g., Agostinelli, Sherman, Fazio, \& Hearst, 1986; Rensink, 1998, 2004). However, this had not previously been evidenced in the free-viewing conditions of CVS, demonstrating that uncertainty can also occur when observers scrutinize objects without constraint.

It is well documented that search slopes vary with the ease with which targets can be detected among distractors, but the present study also demonstrates that the speed with which targets can be identified following detection also contributes to reaction time. In the present experiments, verification/identification processes were seen to take longer depending on the difficulty of the discrimination, despite search alighting on the targets after the same number of fixations. This shows the clear separation between the time taken to search through the display (as would be indicated by traditional search slopes) and the time taken to discriminate a target (as would be indicated by the intercept of a search function). This fact should be considered when one compares search for different object features, 
particularly in change blindness experiments interested in the ease with which different change types can be detected. In highlighting the difference in these stages, the present results contribute to the growing number of studies that recognize the value of eye movements in revealing the processes that characterize search as they unfold over time (see, e.g., Scinto et al., 1986; Zelinsky, Rao, Hayhoe, \& Ballard, 1997).

\section{REFERENCES}

Agostinelli, G., Sherman, S. J., Fazio, R. H., \& Hearst, E. S. (1986). Detecting and identifying change: Additions versus deletions. Journal of Experimental Psychology: Human Perception \& Performance, 12, 445-454.

AnTES, J. (1974). The time course of picture viewing. Journal of Experimental Psychology, 103, 62-70.

Ballard, D., Hayhoe, M., \& Pelz, J. (1995). Memory representations in natural tasks. Journal of Cognitive Neuroscience, 7, 66-80.

Buswell, G. T. (1935). How people look at pictures: A study of the psychology of perception in art. Chicago: University of Chicago Press.

Gould, J. D. (1967). Pattern recognition and eye-movement parameters. Perception \& Psychophysics, 2, 399-407.

Gould, J. D. (1973). Eye movements during visual search and memory search. Journal of Experimental Psychology, 98, 184-195.

Gould, J. D., \& PeEPLes, D. R. (1970). Eye movements during visual search and discrimination of meaningless, symbol, and object patterns. Journal of Experimental Psychology, 85, 51-55.

Henderson, J. M., \& Hollingworth, A. (2003). Eye movements and visual memory: Detecting changes to saccade targets in scenes. Perception \& Psychophysics, 65, 58-71.

Henderson, J. M., Williams, C. C., Castelhano, M. S., \& Falk, R. J. (2003). Eye movements and picture processing during recognition. Perception \& Psychophysics, 65, 725-734.

Hollingworth, A., \& Henderson, J. M. (2002). Accurate visual memory for previously attended objects in natural scenes. Journal of Experimental Psychology: Human Perception \& Performance, 28, 113-136.

Hollingworth, A., Schrock, G., \& Henderson, J. M. (2001). Change detection in the flicker paradigm: The role of fixation position within the scene. Memory \& Cognition, 29, 296-304.

Hooge, I. T. C., \& ERKelens, C. J. (1996). Control of fixation duration in a simple search task. Perception \& Psychophysics, 58, 969-976.

Hooge, I. T. C., \& ERKelens, C. J. (1998). Adjustment of fixation duration in visual search. Vision Research, 38, 1295-1302.

IRWIN, D. E. (1991). Information integration across saccadic eye movements. Cognitive Psychology, 23, 420-456.

IRWIN, D. E., \& ZeLINSKY, G. J. (2002). Eye movements and scene perception: Memory for things observed. Perception \& Psychophysics, 64, $882-895$

JACOBS, A. M. (1986). Eye-movement control in visual search: How direct is visual span control? Perception \& Psychophysics, 39, 47-58.

JACOBS, A. M., \& O'REgAN, J. K. (1987). Spatial and/or temporal adjustments of scanning behavior to visibility changes. Acta Psychologica, $\mathbf{6 5}, 133-146$

Just, M. A., \& Carpenter, P. A. (1976). Eye fixations and cognitive processes. Cognitive Psychology, 8, 441-480.

Karpov, B. A., LuRIa, A. R., \& Yarbus, A. L. (1968). Disturbances of the structure of active perception in lesions of the posterior and anterior regions of the brain. Neuropsychologia, 6, 157-166.

LofTus, G. (1972). Eye fixations and recognition memory for pictures. Cognitive Psychology, 3, 525-551.

Matin, E. (1974). Saccadic suppression: A review and analysis. Psychological Bulletin, 81, 899-917.

McConkie, G. W., \& Currie, C. B. (1996). Visual stability across saccades while viewing complex pictures. Journal of Experimental Psychology: Human Perception \& Performance, 22, 563-581.
Melcher, D., \& Kowler, E. (2001). Visual scene memory and the guidance of saccadic eye movements. Vision Research, 41, 3579-3611.

Nelson, W. W., \& Loftus, G. (1980). The functional visual field during picture viewing. Journal of Experimental Psychology: Human Learning \& Memory, 6, 391-399.

O’REgan, J. K. (1992). Solving the "real" mysteries of visual perception: The world as an outside memory. Canadian Journal of Psychology, 46, 461-488.

PARKER, R. E. (1978). Picture processing during recognition. Journal of Experimental Psychology: Human Perception \& Performance, 4, 284-293.

PHILLIPS, W. A. (1974). On the distinction between sensory storage and short-term visual memory. Perception \& Psychophysics, 16, 283290.

Pomplun, M., Reingold, E. M., \& Shen, J. (2001). Investigating the visual span in comparative search: The effects of task difficulty and divided attention. Cognition, 81, B57-B67.

Pomplun, M., \& RitTer, H. (1999). A three-level model of comparative visual search. In M. Hahn \& S. C. Stoness (Eds.), Proceedings of the Twenty-First Annual Conference of the Cognitive Science Society (pp. 543-548). Mahwah, NJ: Erlbaum.

Pomplun, M., Sichelschmidt, L., Wagner, K., Clermont, T., RickHeIT, G., \& RitTer, H. (2001). Comparative visual search: A difference that makes a difference. Cognitive Science, 25, 3-36.

RAYNER, K. (1998). Eye movements in reading and information processing: 20 years of research. Psychological Bulletin, 124, 372-422.

ReNsinK, R. A. (1998). Mindsight: Visual sensing without seeing [Abstract]. Investigative Ophthalmology \& Visual Science, 39, 631.

RensinK, R. A. (2002). Change detection. Annual Review of Psychology, $\mathbf{5 3}, 245-277$.

Rensink, R. A. (2004). Visual sensing without seeing. Psychological Science, 15, 27-32.

Rensink, R. A., O'Regan, J. K., \& Clark, J. J. (1997). To see or not to see: The need for attention to perceive changes in scenes. Psychological Science, 8, 368-373.

SCInto, L. F., Pillalamarri, R., \& Karsh, R. (1986). Cognitive strategies for visual search. Acta Psychologica, 62, 263-292.

SHEPARD, R. N. (1967). Recognition memory for words, sentences, and pictures. Journal of Verbal Learning \& Verbal Behavior, 6, 155-163.

SHORE, D. I., \& KLEIN, R. M. (2000). The effects of scene inversion on change blindness. Journal of General Psychology, 127, 27-43.

Simons, D. J. (1996). In sight, out of mind: When object representations fail. Psychological Science, 7, 301-305.

Simons, D. J. (2000). Current approaches to change blindness. Visual Cognition, 7, 1-15.

Standing, L., Conezio, J., \& Haber, R. N. (1970). Perception and memory for pictures: Single-trial learning of 2500 visual stimuli. Psychonomic Science, 19, 73-74.

Underwood, G., Chapman, P., Berger, Z., \& Crundall, D. (2003). Driving experience, attentional focusing, and the recall of recently inspected events. Transportation Research Part F, 6, 289-304.

UnDERWOOD, G., JeBbetT, L., \& RoberTs, K. (2004). Inspecting pictures for information to verify a sentence: Eye movements in general encoding and focused search. Quarterly Journal of Experimental Psychology, 57A, 165-182.

YARBUS, A. L. (1967). Eye movements and vision. New York: Plenum.

ZeLinsKy, G. J. (2001). Eye movements during change detection: Implications for search constraints, memory limitations, and scanning strategies. Perception \& Psychophysics, 63, 209-225.

Zelinsky, G. J., RaO, R. P. N., Hayhoe, M. M., \& Ballard, D. H. (1997). Eye movements reveal the spatiotemporal dynamics of visual search. Psychological Science, 8, 448-453.

\section{NOTE}

1. We thank an anonymous reviewer for this suggestion.

(Manuscript received December 12, 2003; revision accepted for publication December 14, 2004.) 